\section{OPEN ACCESS}

Edited by:

Moshe Bensimon,

Bar-llan University, Israel

Reviewed by:

Amy L. Milton,

University of Cambridge,

United Kingdom

Theresa Friederike Wechsler,

University of Regensburg, Germany

*Correspondence:

Thiemo Knaust

thiemo1knaust@bundeswehr.org

Holger Schulz

schulz@uke.de

Specialty section:

This article was submitted to

Psychology for Clinical Settings,

a section of the journal

Frontiers in Psychology

Received: 15 May 2020 Accepted: 30 September 2020

Published: 13 November 2020

Citation:

Knaust T, Felnhofer $A$ Kothgassner OD, Höllmer $H$,

Gorzka R-J and Schulz H (2020) Virtual Trauma Interventions for the Treatment of Post-traumatic Stress

Disorders: A Scoping Review.

Front. Psychol. 11:562506 doi: $10.3389 /$ fpsyg.2020.562506

\title{
Virtual Trauma Interventions for the Treatment of Post-traumatic Stress Disorders: A Scoping Review
}

\author{
Thiemo Knaust ${ }^{1 *}$, Anna Felnhofer ${ }^{2}$, Oswald D. Kothgassner ${ }^{3}$, Helge Höllmer ${ }^{1}$, \\ Robert-Jacek Gorzka ${ }^{4}$ and Holger Schulz ${ }^{5 *}$ \\ ${ }^{1}$ Center for Mental Health, Bundeswehr Hospital Hamburg, Hamburg, Germany, ${ }^{2}$ Department of Pediatrics and Adolescent \\ Medicine, Medical University of Vienna, Vienna, Austria, ${ }^{3}$ Department of Child and Adolescent Psychiatry, Medical University \\ of Vienna, Vienna, Austria, ${ }^{4}$ Department of Applied Military and Operational Psychology, Military Police Command, Hanover, \\ Germany, ${ }^{5}$ Department of Medical Psychology, University Hospital Hamburg-Eppendorf, Hamburg, Germany
}

Some post-traumatic stress disorder (PTSD) patients do not benefit from imaginal exposure therapy. One possible approach to reach such patients are virtual trauma interventions. Herein, a qualitative scoping review was conducted. Different types of virtual trauma exposure interventions were identified. For each type of virtual trauma exposure interventions it was examined in detail: (1) which in sensu trauma exposure approach serves as therapeutic framework, how it was transferred into virtual reality, and if it was manualized; (2) which hardware and software were used; (3) whether the influence of spatial and social presence on the efficacy of virtual trauma interventions have been measured, and (4) whether the efficacy of virtual trauma interventions for PTSD patients having imagination difficulties was evaluated. These research questions were analyzed qualitatively. Accordingly, an extensive literature search was conducted using the databases Web of Science, PsycINFO, LIVIVO, PTSDpubs, and PubMed for scientific articles published between January 2013 and July 2020. Only studies aimed to reduce PTSD symptoms using virtual trauma interventions were included. The literature search was not limited to a specific study design, treatment/intervention method, or a minimum sample size. Eighteen studies were identified, which reported three different virtual trauma intervention approaches, namely, virtual reality exposure therapy (VRET), multi-modular motion-assisted memory desensitization and reconsolidation (3MDR), and action-centered exposure therapy (ACET). Seven randomized controlled trials (RCTs), two pilot studies, and one case study were focused on VRET; while two RCTs, one pilot study, and three case studies focused on 3MDR, and two case studies on ACET. Regarding the first research question (1), the results show that VRET is based on prolonged exposure, aiming for a virtual re-creation of the patient's traumatic recounting. Several treatment protocols exist for VRET. 3MDR is based on eye movement desensitization and reprocessing, aiming to reduce the patient's avoidance behavior. In 3MDR patients walk toward individualized trauma-related symbolic images in a cave automatic virtual environment (CAVE). One treatment protocol exists for 3MDR. ACET is based on the inhibitory learning theory, aiming for active interactions with a virtual trauma-associated environment to alter the anxiety structure through new secondary 
inhibitory learning. One treatment protocol exists for ACET. For the second research question (2), the results indicate that all VRET studies used head-mounted displays (HMDs) with a virtual version of the Iraq/Afghanistan or the World Trade Center attacks, while 3MDR studies utilized two different versions of a CAVE with personalized trauma-related images, and the ACET studies used HMDs with virtual street scenarios. For the third research question (3), the results demonstrate that the influence of spatial or social presence on the efficacy of virtual trauma interventions was not examined in any of the included studies. Similarly, for the fourth research question (4), the results show that empirical evidence for the efficacy of virtual trauma interventions on PTSD patients having imagination difficulties was lacking. Therefore, such empirical studies are needed to fill these research gaps.

Keywords: virtual reality exposure therapy, VRET, multi-modular motion-assisted memory desensitization and reconsolidation, 3MDR, action-centered exposure therapy, ACET, virtual trauma interventions, PTSD

\section{INTRODUCTION}

The main category of in sensu confrontation (or imaginal exposure) includes different interventions e.g., prolonged exposure (PE; Foa et al., 2007, 2019), eye movement desensitization and reprocessing (EMDR; Shapiro, 1989, 2018), or imagery rescripting and reprocessing therapy (IRRT, Schmucker and Köster, 2019). For the treatment of posttraumatic stress disorder (PTSD), PE and EMDR, are ranked as the oldest, best-examined, and most effective techniques (Foa et al., 2007; Powers et al., 2010; National Institute for Health Care Excellence, 2018; Schäfer et al., 2019). Despite the large empirical support for the efficacy of PE and EMDR not every patient benefits from these therapeutic approaches (examples for PE: Jaycox et al., 1998; Marks et al., 1998; Taylor et al., 2003; Foa et al., 2005, 2018; McDonagh et al., 2005; Schnurr et al., 2007; Pacella et al., 2011; Acierno et al., 2016; examples for EMDR: Marcus et al., 1997; Devilly et al., 1998; Power et al., 2002; Karatzias et al., 2011; Acarturk et al., 2016; Carletto et al., 2016). A recent meta-analysis showed a mean attrition rate of $22 \%$ for PE (included studies: $k=22$ ) and $18 \%$ for EMDR (included studies: $k=21$, Lewis et al., 2020). Furthermore, it remains unclear how many patients benefit from PE and EMDR in terms of remission and response rate. In some efficacy studies on $\mathrm{PE}$, a remission rate of approximately $75 \%$ was reported (Marks et al., 1998; Taylor et al., 2003), whereas in other studies, it was approximately 40\% (Foa et al., 1991; McDonagh et al., 2005; Schnurr et al., 2007; Fonzo et al., 2017). In a more recent study, the efficacy of mass (all sessions in 2 weeks, $N=110$ ) was compared with spaced PE (all sessions in 8-15 weeks, $N=$ 109 ), and remission rates of $54 \%$ and $51 \%$ were found (Foa et al., 2018). A similar pattern was observed for EMDR (Marcus et al., 1997; Carlson et al., 1998; Devilly and Spence, 1999; Högberg et al., 2007; Capezzani et al., 2013; Acarturk et al., 2016; Carletto et al., 2016). Here, efficacy studies revealed a remission rate of 36\% (Devilly and Spence, 1999)-90\% (Capezzani et al., 2013). This type of heterogeneity was also shown for the response rate of PE and EMDR (Foa et al., 1991; Devilly and Spence, 1999; Power et al., 2002; Schnurr et al., 2007; Asukai et al., 2010;
Karatzias et al., 2011; Rauch et al., 2014). In efficacy studies on PE and EMDR, response rates of 40-90\% (Foa et al., 1991; Rauch et al., 2014) and 27\% (Devilly and Spence, 1999)-76\% (Karatzias et al., 2011), respectively, were revealed (Supplementary Table 1 provides a more detailed overview of the cited studies and their operationalization of attrition, remission and response rate).

The reasons for the attrition, response and remission rates are multifactorial and not completely understood (Lewis et al., 2020). Some authors argue that for example, the traumatype (Steenkamp et al., 2015; Wagenmans et al., 2018), psychiatric comorbidities (Van Minnen et al., 2012), high avoidance (Zoellner et al., 2011; Hundt et al., 2018; Van Gelderen et al., 2020) or insufficient imagination abilities (Jaycox et al., 1998) may explain whether patients can benefit from imaginal techniques. However, further work is required to verify these assumptions.

Nevertheless, this introductory overview provides evidence for the argument that not all patients benefit from PE and EMDR. To allow successful treatment for patients who are unable to benefit from classic trauma-focused guidelines, it is particularly important to use and examine the variety of treatment methods available in the context of psychotraumatology (Rizzo and Shilling, 2017; Carl et al., 2018; Kothgassner et al., 2019).

\section{Trauma Therapy and Virtual Reality}

In general, trauma exposure can be explained by the emotional processing theory (Foa and Kozak, 1986), which presumes that the PTSD symptoms are based on pathological fear structures that are activated when patients are confronted with traumarelevant information (Hembree et al., 2003). A reduction of symptoms requires a modification of the affective memory, enabling emotional processing such as the trauma-related information no longer evokes fear (Foa and Kozak, 1986). Therefore, imaginal exposure strives for mental engagement with the fear structure through confrontations (in sensu or in vivo) to achieve the habituation and extinction of an anxious reaction (Foa and Kozak, 1986; Foa et al., 2007). This can be practically achieved by the recounting of the patient's traumatic experience guided and encouraged by a therapist to imagine, narrate, and 
emotionally process the traumatic event (Foa et al., 2007; Leaman et al., 2013). However, this is particularly difficult for some patients, especially for those who cannot visualize the traumatic event or are not willing or prepared to do so because of memory gaps or severe avoidance behavior (e.g., an emotional defense mechanism) (Difede et al., 2007; Kehle-Forbes et al., 2016; Rizzo and Shilling, 2017; Loucks et al., 2018; Shulman et al., 2019). This can lead to a (premature) termination of treatment or to a less pronounced emotional reactivity when reporting the trauma, which negatively affects the treatment success (Jaycox et al., 1998; Difede et al., 2007; Foa et al., 2007; Cukor et al., 2015).

Therefore, studies have examined the possibility of incorporating virtual reality (VR) in trauma therapy to reach these patients (Rothbaum et al., 2001; Difede et al., 2007; Leaman et al., 2013; Vermetten et al., 2013; Cukor et al., 2015). There are various approaches to integrating VR in trauma therapy (Leaman et al., 2013; Vermetten et al., 2013). One kind of virtual trauma intervention is virtual reality exposure therapy (VRET) (Rothbaum et al., 1999, 2001). In VRET, head-mounted displays (HMDs) with preprogrammed virtual scenarios/environments are used to recreate the patients' traumatic experiences (Leaman et al., 2013; Rizzo and Shilling, 2017). Another way to use VR in trauma therapy is multi-modular motion-assisted memory desensitization and reconsolidation (3MDR) (Vermetten et al., 2013). 3MDR combines a treadmill with computer automatic virtual environments (CAVEs) to reduce PTSD patients' avoidance behavior (Vermetten et al., 2013; Van Gelderen et al., 2018). In $3 \mathrm{MDR}$, it is assumed that walking toward a traumarelated symbolic representation of the patients' traumatic experience in a CAVE decreases avoidance behavior, increases therapy adherence, and reduces PTSD symptoms (Van Gelderen et al., 2018). The virtual environment can be customized through images selected by the patients (Van Gelderen et al., 2020).

From a cost-efficiency perspective, both approaches offer advantages and disadvantages. For example, an advantage of VRET is that the costs for HMDs are now less expensive and becoming more affordable, which makes VRET more obtainable in a clinical setting (Rizzo and Shilling, 2017; Kothgassner et al., 2019). However, a disadvantage of VRET is the high software cost (Rizzo and Shilling, 2017). To implement VRET in a clinical setting using IT, engineers program a virtual environment with preprogrammed and editable scenarios (Rizzo et al., 2005; Rizzo and Shilling, 2017). These scenarios are necessary tools that the therapist uses to customize the virtual environment based on the patient's recounting (Leaman et al., 2013). The generation of these scenarios is expensive and time-consuming. For example, Rizzo and Shilling (2017) mentioned a development time of 3 years. By contrast, for $3 \mathrm{MDR}$, the hardware costs, for example, using CAVE, are still relatively high-ranging from $\$ 50,000-$ 100,000 (Borrego et al., 2015; Coburn et al., 2017). Additionally, to conduct a $3 \mathrm{MDR}$ session, a technical operator and therapists are needed, which increases personnel costs (Vermetten et al., 2013; Van Gelderen et al., 2020). However, there is empirical evidence that $3 \mathrm{MDR}$ is a successful treatment for patients who cannot benefit from classic trauma-focused guidelines, which can reduce long-term therapy costs (Bisson et al., 2020; Van Gelderen et al., 2020). Nevertheless, the cost-efficiency aspect of virtual trauma intervention is not satisfactorily answered through research.

In addition to the general cost-efficiency debate, the mostexamined virtual trauma intervention is VRET (Carl et al., 2018; Deng et al., 2019; Kothgassner et al., 2019). VRET uses HMD to offer a multi-sensory, anxiety-provoking, and traumaspecific virtual environment, which can be adjusted individually to the patients' own traumatic experience by adding or removing trauma-specific stimuli, leading to a controllable, repeatable, and emotionally engaging virtual trauma environment (Leaman et al., 2013; Ecrepont et al., 2016; Rizzo and Koenig, 2017; Rizzo and Shilling, 2017). This type of virtual re-creation is presumed to be helpful for patients with imagination difficulties to gain better access to their trauma-associated stimuli and memories (Jaycox et al., 1998; Difede et al., 2007; Leaman et al., 2013; Cukor et al., 2015; Rizzo and Shilling, 2017). In addition, VRET offers the advantage of gradually generating a standardized exposure and repeating it immediately ad infinitum (Leaman et al., 2013; Rizzo and Shilling, 2017; Kothgassner et al., 2019).

Several studies have been conducted to evaluate the efficacy of using VRET for PTSD (Difede et al., 2007; Ready et al., 2010; McLay et al., 2011; Miyahira et al., 2012). These early efficacy studies have revealed that VRET is superior to waitlist conditions with a medium to large effect size (Difede et al., 2007; Miyahira et al., 2012), although no significant differences in active controls have been shown e.g., present-centered therapy (PCT; Ready et al., 2010) or treatment as usual (TAU; McLay et al., 2011). Three recent meta-analyses have summarized the previous efficacy studies of VRET for PTSD (Carl et al., 2018; Deng et al., 2019; Kothgassner et al., 2019). Carl et al. (2018) identified five studies in which VRET was compared with the waitlist condition. The meta-analysis showed that VRET is superior to a waitlist with a medium effect size (hedge's $g=0.59,95 \%$ CI $[0.26,0.92]$ ). Similarly, Deng et al. (2019) found that VRET is superior to inactive controls (hedge's $g=0.56,95 \%$ CI $[0.27,0.86]$, included studies: $k=5, N=175$ ), although there was no significant effect compared to the active controls (e.g., PE) (hedge's $g=0.01,95 \%$ CI $[-0.41,0.44]$, included studies: $k=6, N=239)$. Additionally, Kothgassner et al. (2019) revealed that VRET is superior to the waitlist control (hedge's $g=0.56,95 \%$ CI [0.27, 0.86], included studies: $\left.k=4, N_{V R E T}=54, N_{\text {Waitlist }}=68\right)$, although there was no significant difference shown between VRET and the active controls (hedge's $g=0.25,95 \%$ CI $[-0.28,0.79]$, included studies: $\left.k=6, N_{V R E T}=100, \mathrm{~N}_{\text {activecontrols }}=104\right)$.

These meta-analyses identified only two (Deng et al., 2019) and three studies (Kothgassner et al., 2019), which compared VRET to in sensu confrontation. Therefore, no meta-analyses so far have been conducted comparing VRET exclusively to in sensu confrontation (Carl et al., 2018; Deng et al., 2019; Kothgassner et al., 2019). Instead, in sensu confrontation was subsumed with different interventions (e.g., PCT) to active controls. Herein, the results show that VRET is superior to a waitlist, although no significant difference was shown between the active controls. However, recent reviews and meta-analyses have also indicated that the results have yet to be sufficiently confirmed statistically (Carl et al., 2018; Deng et al., 2019; Kothgassner et al., 2019). There have been very few randomized controlled studies (RCTs), 
and mostly small sample sizes have been used in the RCTs included in the meta-analyses (Carl et al., 2018; Deng et al., 2019; Kothgassner et al., 2019). Furthermore, these meta-analyses focused exclusively on VRET, which is one specific kind of virtual trauma exposure (Carl et al., 2018; Deng et al., 2019; Kothgassner et al., 2019). Therefore, less is known about other approaches that use VR in trauma therapy.

Except for the relatively well-established efficacy of VRET, the exact mechanism of virtual trauma exposure has yet to be fully clarified. It has generally been hypothesized that immersive technology enables the patient to experience a spatial presence (also known as the sense of being there in an artificial environment) (Wirth et al., 2007; Hartmann et al., 2016). Spatial presence, in turn, is presumed to be an essential precondition for activating a trauma-associated anxiety structure during VRET (Leaman et al., 2013; Vermetten et al., 2013; Cukor et al., 2015). In addition to spatial presence, it is also conceivable that social presence may influence the efficacy of VRET, particularly for interpersonal traumas such as sexual abuse or combat-related trauma (Ling et al., 2014; Oh et al., 2018). Social presence is described as "the sense of being with another" (Biocca et al., 2003, p. 456). It is essential for the user to experience a real social interaction with artificial intelligence-designed avatars (Lee et al., 2006; Oh et al., 2018). Without social presence, the sensation of being with another person in a virtual environment is "merely experienced as an artificial entity and not as a social being" (Oh et al., 2018, p. 2).

Moreover, in different studies in which VRET was used for other anxiety disorders, spatial presence was presumed to be an important factor influencing treatment success (Ling et al., 2014; Botella et al., 2017). For instance, Price and Anderson (2007) examined whether spatial presence during a virtual confrontation for patients with a fear of flying mediates treatment success. They showed that higher levels of spatial presence are related to higher in-session anxiety. However, they did not find that higher spatial presence is related to treatment success. Two more recent systematic reviews concluded that there is a significant correlation between spatial presence and anxiety during virtual confrontations (e.g., acrophobia: $r=.35, p<0.001$, included correlations: $k=14$; fear of animals: $r=.50, p<0.001$, included correlations: $k=12$ and fear of flying: $r=.50, p<0.001$, included correlations: $k=12$; Ling et al., 2014, p. 6) (Ling et al., 2014; Botella et al., 2017). However, it remains unclear whether the sense of presence is a precondition for treatment success, and the causal direction of the mean correlation between spatial presence and rated anxiety is still undetermined (Ling et al., 2014; Botella et al., 2017).

\section{Objectives of the Current Review}

Prior reviews and meta-analyses have examined the efficacy of VRET in comparison to an active or inactive control group in anxiety disorders and PTSD (Botella et al., 2015; Carl et al., 2018; Deng et al., 2019; Kothgassner et al., 2019). It has yet to be summarized which other virtual trauma exposure intervention approaches exists, besides VRET. In more detail, it should be examined which in sensu trauma exposure approach serves as therapeutic framework for each identified type of virtual trauma exposure intervention, how it was transferred into VR, and if and how it is manualized. Furthermore, it is unclear which hardware and software are used for virtual trauma interventions and whether the experience of spatial or social presence is empirically assessed and influences the efficacy of a virtual confrontation. Finally, it is assumed that virtual trauma interventions are particularly effective for PTSD patients with imagination difficulties (Difede et al., 2007; Rizzo and Shilling, 2017; Loucks et al., 2018). However, it remains unclear whether previous studies have examined this assumption empirically. Therefore, it should be evaluated whether previous studies have empirically examined this assumption.

To identify as many virtual trauma interventions as possible, we conducted a qualitative scoping review. The literature search was not limited to a specific study design, treatment/intervention method or a minimal sample size. However, we only included studies with the goal of reducing PTSD symptoms with virtual trauma confrontations. More precisely, we defined the main characteristic of virtual trauma intervention to be an immersive technology. Technology is defined as immersive if it delivers "an inclusive, extensive, surrounding, and vivid illusion of reality" (Slater and Wilbur, 1997, p. 604). In general, HMDs and cave automatic virtual environments (CAVEs) meet these criteria and are considered immersive technologies (Cipresso et al., 2018, p. 4).

In conclusion, the overarching goal of the present review is to identify different types of virtual trauma exposure interventions and to examine for each identified type of virtual intervention (1) which in sensu trauma exposure approach serves as therapeutic framework for this type of virtual trauma exposure intervention, how it was transferred into VR (kind of virtual trauma exposure environment and procedure of virtual trauma exposure intervention), and if it is manualized; (2) assess which hardware and software are used; (3) examine whether the influence of spatial and social presence on the efficacy of virtual trauma interventions has been measured, and (4) evaluate whether these virtual trauma interventions are particularly effective for PTSD patients with imagination difficulties.

\section{METHODS}

\section{Scoping Review}

We chose a scoping review with a qualitative synthesis as a suitable type of review particularly with regards to the accelerated development of immersive technology and the currently existing research gaps for virtual trauma interventions. The present scoping review followed current guidelines for scoping reviews (Pham et al., 2014; Peters et al., 2015; Tricco et al., 2018).

\section{Search Strategy}

We performed a systematic literature search using the electronic databases Web of Science, PsycINFO, LIVIVO, PTSDpubs, and PubMed with the following search terms in German and English: ("VR" OR "Virtual Reality" OR "Virtuelle Realität") AND ("PTBS” OR "PTSD” OR "Posttraumatische Belastungsstörung” OR "Posttraumatic Stress Disorder" OR "Post-traumatic Stress Disorder"). In 2013 the popularity of VR technology increased, 
based on the technological advances (the release of the Developer Kit of the Oculus Rift) (Luckerson, 2014; Cipresso et al., 2018). To increase the likelihood of identifying virtual trauma interventions, which should use modern VR technology, we conducted the literature search starting from January 2013 to August 2018 and performed an update of our search including the period from August 2018 to July 2020.

\section{Inclusion and Exclusion Criteria}

Based on our research questions, we a priori defined the following inclusion and exclusion criteria: we included (1) studies which used any type of virtual trauma intervention with the goal to reduce PTSD symptoms; (2) studies which used immersive technology; (3) studies which used virtual exposure; (4) research in which PTSD was diagnosed according to ICD-10, DSM IV, DSM IV-R or DSM-5; (5) studies which examined patients between the ages of 18 and 65 years; (6) studies which were published from 2013 onwards, and (7) peer-reviewed articles.

We excluded (1) studies which used virtual trauma interventions for other purposes than for the treatment of PTSD $^{1}$ e.g., basic research purpose and (2) studies which did not include original data (e.g., book chapters, reviews, study protocols, comments, etc. $)^{2}$.

\section{Course of Evaluation of Suitability}

In the first step, we exported the title, abstract, year and authors of the identified literature into an Excel table and then removed duplicates. Two independent reviewers (VT, PS) read the titles and abstracts of each study. In case of disagreements, another reviewer (TK) was consulted, who decided whether the study met or did not meet the inclusion criteria. The PRISMA flowchart shows exclusions of studies at each stage of the process (see Figure 1).

\section{Data Extraction}

A data extraction table was generated to answer the research questions. For the first research question, we extracted the type of virtual trauma exposure (e.g., VRET), the trauma-focused in sensu framework (e.g., PE or EMDR), how it was transferred into VR (e.g., virtual re-creation), and if it was manualized. If the studies used a manualized treatment protocol, we extracted in detail: the number of sessions and period of treatment time, the number and period of virtual exposure (in particular, it was examined whether there was a specific time limit of virtual exposure or did the studies conducted the virtual exposure until within-session habituation was attained), whether the treatment was supplemented with medication (if so, which medication was used), and whether at-home in vivo exposure exercises were included in the treatment protocol. For the second research question, the hardware and software used for the virtual trauma interventions were extracted. For the third research question, it was extracted whether and how the influence of spatial or

\footnotetext{
${ }^{1}$ This includes, e.g., studies that examined virtual trauma exposure focusing on diagnostic aspects (Ridout et al., 2017; Van't Wout et al., 2017; Maples-Keller et al., 2019).

${ }^{2}$ In reviews and book chapters, the references were examined by hand to identify further publications.
}

social presence on the efficacy of virtual trauma interventions was measured. For the last one, we extracted whether and how the evaluation of efficacy for PTSD patients with imaginary problems were measured.

In addition, general descriptive variables were extracted. These included: authors, title, year, country of publication, the instrument for PTSD diagnosis (e.g., Diagnostic and Statistical Manual of Mental Disorders 5th Edition, DSM-5), the primary outcome variable, the time points of measurements (e.g., post-treatment, 3-month-follow-up, etc.), the effect sizes (if the authors did not reported the effect sizes, we extracted means, standard deviations and sample sizes for the respective studies. To recalculate the effect sizes we followed the procedure by Hedges and Olkin, 2014), the study design, the sample characteristic (military or civilian sample) and size, the drop-out rate, the average age of the sample, the gender distribution of the sample, and the trauma type. Furthermore, we extracted the objectives and main results of each included study.

\section{Data Synthesis}

We provide a general overview of studies on virtual trauma intervention by calculating the overall descriptive statistics of the included studies (e.g., we calculated the sum of male patients over all the studies to obtain an overview of the gender distributions across all the studies). To analyze the research questions, we grouped the studies according to the identified types of virtual trauma interventions. For the first research question, we identified the in sensu trauma exposures that worked as therapeutic frameworks and qualitatively summarized how they were transferred to VR. To explore and compare the treatment protocols in further detail (the third part of the first research question), we tabulated and sorted the included studies according to the number of therapy sessions, time of each session, overall period of time, number of virtual exposures and duration of each. Thus, we examined whether the identified types of virtual trauma intervention followed the same procedure. After tabulating and sorting, we grouped the studies that were sufficiently similar and calculated descriptive statistics (percentage). Furthermore, we determined whether the researchers supplemented their treatment protocols with medication and at-home in vivo exercises. To summarize these results, we also calculated percentages. For the second research question, we calculated the percentage of the hardware and software used. The same procedure was followed for the third and fourth research questions.

\section{RESULTS}

\section{General Overview}

We identified a total of 805 articles, of which 18 met the inclusion criteria. Of the 18 studies, nine (50\%) (Difede et al., 2013; Rothbaum et al., 2014; Reger et al., 2016; Beidel et al., 2017a; McLay et al., 2017; Maples-Keller et al., 2018; Van't Wout et al., 2018; Bisson et al., 2020; Van Gelderen et al., 2020) were RCTs with a total of 483 patients (317 received VR treatment, 100 received an active treatment and 66 allocated to waitlist). Six 


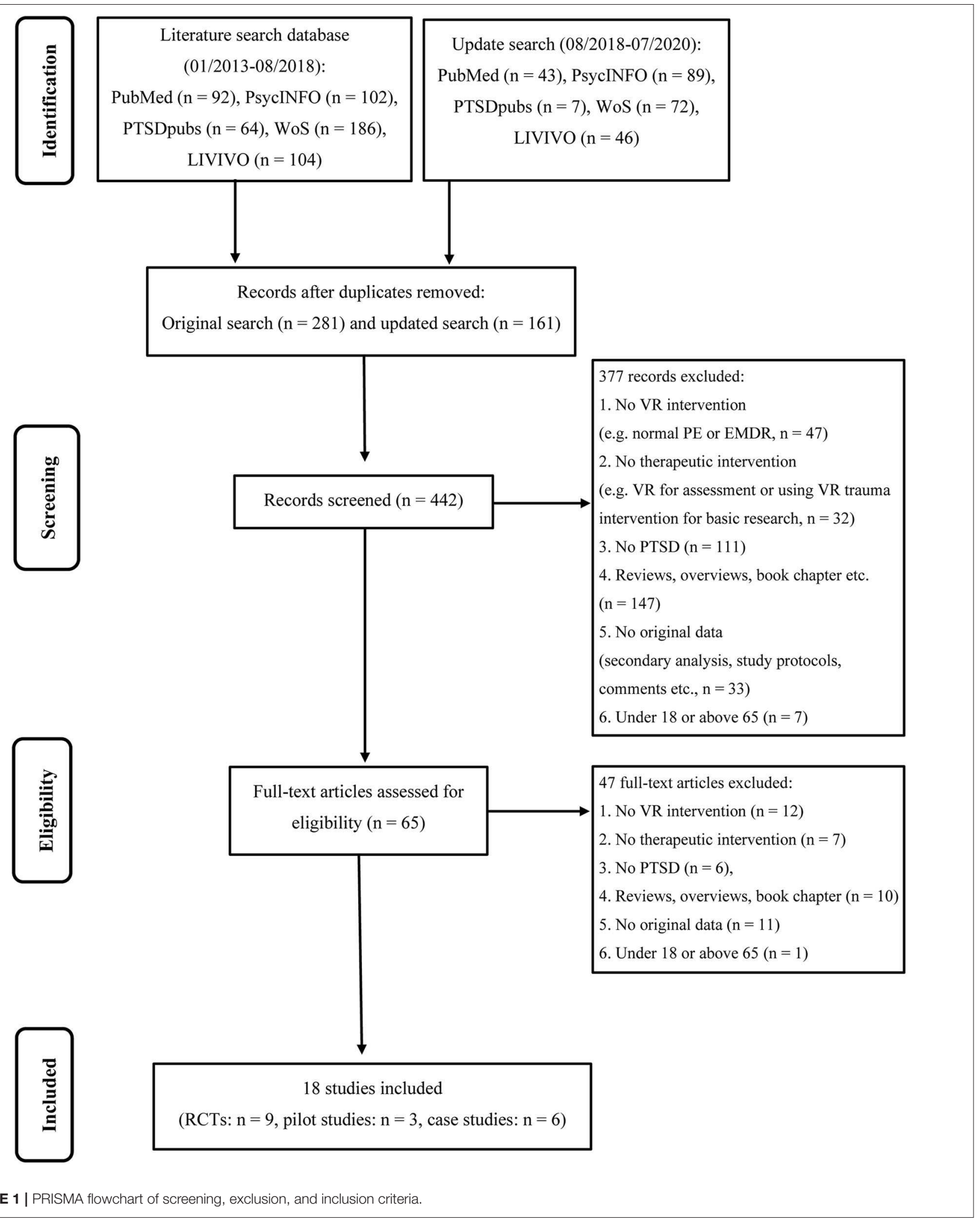

(33.3\%) (Vermetten et al., 2013; Arens, 2014; Kengne et al., 2018; Menelas et al., 2018; Nijdam and Vermetten, 2018; Van Gelderen et al., 2018) were case studies examined a total of 10 patients and three (16.6\%) (Beidel et al., 2017b; Jetly et al., 2017; Loucks et al.,
2018) were non-randomized single-arm trials subsumed to pilot studies with a total of 116 patients.

Ten studies (55.5\%) (Difede et al., 2013; Arens, 2014; Rothbaum et al., 2014; Reger et al., 2016; Beidel et al., 2017a,b; 
McLay et al., 2017; Loucks et al., 2018; Maples-Keller et al., 2018; Van't Wout et al., 2018) were conducted in North America, three (16.6\%) (Jetly et al., 2017; Kengne et al., 2018; Menelas et al., 2018) in Canada, four in the Netherlands (22.2\%) (Vermetten et al., 2013; Nijdam and Vermetten, 2018; Van Gelderen et al., $2018,2020)$ and one in Wales, United Kingdom (5.5\%) (Bisson et al., 2020). Patients were predominantly male (92.7\%), and the mean age ranged from 29 (Reger et al., 2016) - 51 (Nijdam and Vermetten, 2018). 15 (83.3\%) studies included soldiers or veterans with combat-related PTSD, with one specializing on PTSD after a military sexual trauma (MST) (Loucks et al., 2018). Two studies (11.1\%) reported results from civilian truck drivers after an accident (Kengne et al., 2018; Menelas et al., 2018) and one $(5.5 \%)$ examined civilians with PTSD related to the attack on the World Trade Center (WTC) (Difede et al., 2013). Table 1 summarizes the descriptive analysis of the 18 articles.

Regarding the main research question, the results indicate that there are three different approaches to a virtual trauma intervention, namely, VRET (Difede et al., 2013; Arens, 2014; Rothbaum et al., 2014; Reger et al., 2016; Beidel et al., 2017a,b; McLay et al., 2017; Loucks et al., 2018; Maples-Keller et al., 2018; Van't Wout et al., 2018), 3MDR (Vermetten et al., 2013; Jetly et al., 2017; Nijdam and Vermetten, 2018; Van Gelderen et al., 2018, 2020; Bisson et al., 2020), and action-centered exposure therapy (ACET) (Kengne et al., 2018; Menelas et al., 2018). The following describes the results of all research questions separated based on the application of VRET, 3MDR, and ACET (see Table 2 for the results of the qualitative analysis for each study and Table 3 for an overview of the results).

\section{Virtual Reality Exposure Therapy (VRET) Therapeutic Framework Transferred Into VRET}

VRET is based on the therapeutic framework of PE. Here, a patient's traumatic experience was administered virtually, and not through an imagination-based approach (Rothbaum et al., 2010; Leaman et al., 2013). Usually, the patients recount their traumatic memories during the VRET study's virtual confrontations and the therapists match the virtual environments to the recounted experiences (Rothbaum et al., 2010). This type of regeneration/virtual re-creation was presumed to enhance the patient access to trauma-associated stimuli and memories (Rothbaum et al., 2010; Leaman et al., 2013). Nine of 10 (90\%) studies included soldiers or veterans with combat-related PTSD, with one specializing on PTSD after an MST (Loucks et al., 2018). One (10\%) study examined civilians with PTSD related to the attack on the WTC (Difede et al., 2013).

In $90 \%$ of VRET studies, virtual Iraq/Afghanistan or an updated version, called Bravemind, has been used (Rizzo et al., $2005,2017)$. These virtual environments include comprehensive prototype scenarios of combat-related PTSD experiences, such as, riding in a Humvee through a desert (Rizzo et al., 2005). Moreover, a clinical interface was integrated into virtual Iraq/Afghanistan and the Bravemind systems (Rizzo et al., 2017), which enabled the therapist to customize the virtual environments in real-time (e.g., daytime duration, weather conditions, and ambient sounds), to match the patient recounted experiences (Leaman et al., 2013; Rizzo et al., 2017). The clinical interface also allows the therapist to add trigger stimuli, such as explosions or gunfire attacks (Rizzo et al., 2005). Typically, this feature was used in later sessions, when the therapist and patient focus on trauma hotspots, i.e., the portions of traumatic memories causing high levels of anxiety and emotional distress (Leaman et al., 2013).

Several treatment protocols for VRET exist (McLay et al., 2011; Leaman et al., 2013; Rothbaum et al., 2014; Reger et al., 2016). Therefore, the actual procedure varied across studies. The treatment protocol began with one to two preparatory sessions in $70 \%$ of the VRET studies (Difede et al., 2013 ${ }^{3}$; Rothbaum et al., 2014; Reger et al., 2016; McLay et al., 2017; Loucks et al., 2018; Maples-Keller et al., 2018; Van't Wout et al., 2018). Here, the clinician provided an overview of VRET, discussed the treatment duration and adherence, gathered the patient traumatic experience-related information, and presented the rationale of in vivo and virtual exposure. These sessions were followed by five (Rothbaum et al., 2014) to 10 (McLay et al., 2017) 90-min sessions, including 30-45 min for virtual exposure and an approximately 20 -min conversation to support patients in processing their trauma-related notions, thoughts, and feelings.

In contrast, three studies conducted virtual exposure until the patients achieved within-session habituation (Arens, 2014; Beidel et al., 2017a,b). Within-session habituation was operationalized with a $50 \%$ lower anxiety than the session peak. The session peak was measured using a subjective unit of distress (SUD) index, on a scale from 0 to 8 (Arens, 2014; Beidel et al., 2017a,b). Therefore, the first virtual confrontation sessions continued for 90-120 min and the later ones for 15-20 min (Arens, 2014; Beidel et al., 2017a,b). Furthermore, if the patients' distress level did not increase during virtual exposure (between session habituations), the therapists switched to in vivo exposure. Therefore, the therapists used actual places and situations related to the patient's traumatic experience (e.g., crowded places, driving on roads similar to the location of an IED explosion) (Arens, 2014; Beidel et al., 2017a,b).

Seven studies included at-home in vivo exposure exercises to avoided situations (e.g., sleeping with a bathroom door open or sitting with one's back to a doorway) (Difede et al., 2013; Arens, 2014; Reger et al., 2016; Beidel et al., 2017a,b; McLay et al., 2017; Loucks et al., 2018), while three did not (Rothbaum et al., 2014; Maples-Keller et al., 2018; Van't Wout et al., 2018). In summary, the therapists customized the virtual environment to the patient recounting during the virtual exposure in VRET studies. However, due to the heterogeneity in treatment protocols, the generalization of VRET procedures is difficult.

\section{Hardware and Software Used for VRET}

Regarding the second research question, in $80 \%$ of VRET studies (Arens, 2014; Rothbaum et al., 2014; Reger et al., 2016; Beidel et al., 2017a,b; McLay et al., 2017; Loucks et al., 2018; MaplesKeller et al., 2018; Van't Wout et al., 2018), eMaginZ800 HMD (released in 2005), which has a pixel resolution of $800 \times 600$ and a $40^{\circ}$ diagonal field of view for each eye (Rizzo et al., 2005),

\footnotetext{
${ }^{3}$ Difede et al. (2013) also reported that the virtual exposure sessions were conducted until with-session habituation was attained (50\% reduction of the SUD). However, they also set a time limit of exposure (45 min).
} 
TABLE 1 | Descriptive characteristics of $k=18$ included studies.

\begin{tabular}{|c|c|c|c|c|c|c|c|c|}
\hline References & Country & $\begin{array}{l}\text { Instrument } \\
\text { for PTSD } \\
\text { diagnosis }\end{array}$ & $\begin{array}{l}\text { Primary } \\
\text { outcome } \\
\text { variable }\end{array}$ & $\begin{array}{l}\text { Study } \\
\text { design }\end{array}$ & $\begin{array}{l}\text { Sample and } \\
\text { trauma type }\end{array}$ & Participants and drop-out & Intervention & Time points of measurements and main results \\
\hline Arens (2014) & USA & DSM-IV-TR & CAPS & $\begin{array}{l}\text { Case } \\
\text { study }\end{array}$ & $\begin{array}{l}\text { War veteran with } \\
\text { past Iraq and } \\
\text { Afghanistan } \\
\text { deployment. } \\
\text { Combat- } \\
\text { related PTSD }\end{array}$ & $\begin{array}{l}\text { Participant: } N=1 \text {, drop-out: n.a., } \\
\text { age: } 45 \text {, gender: } 1(100 \%) \text { male }\end{array}$ & VRET with TMT & $\begin{array}{l}\text { Measurements: Pre, post, and } 3 \text { month-follow-up } \\
\text { Effect size: n.a. } \\
\text { Summary: Clinically significant decreases in overall PTSD } \\
\text { symptoms. The symptom decrease was maintained at the } \\
3 \text { month-follow-up }\end{array}$ \\
\hline $\begin{array}{l}\text { Beidel et al. } \\
\text { (2017a) }\end{array}$ & USA & DSM-IV & CAPS & $\mathrm{RCT}$ & $\begin{array}{l}\text { War veterans and } \\
\text { active duty } \\
\text { personnel with } \\
\text { past Iraq and } \\
\text { Afghanistan } \\
\text { deployment } \\
\text { Combat-related } \\
\text { PTSD symptoms }\end{array}$ & $\begin{array}{l}\text { Total participants: } N=92 \text {, drop-out: } \\
43(41.3 \%) \text {, mean age: } 35.4 \text {, gender: } \\
86(93.4 \%) \text { males } \\
\text { VR treatment group (VRET with TMT): } \\
n=49, \text { drop-out: } 18(36 \%) \text {, mean } \\
\text { age: } 37.6, \text { gender: } 45(91 \%) \text { males } \\
\text { Active control group (VRET with } \\
\text { psychoeducation): } n=43 \text {, drop-out: } \\
25(58 \%) \text {, mean age: } 33.2 \text {, gender: } \\
41(95 \%) \text { males }\end{array}$ & $\begin{array}{l}\text { VRET with TMT } \\
\text { vs. VRET with } \\
\text { psychoeducation }\end{array}$ & $\begin{array}{l}\text { Measurements: Pre, post, } 3 \text { and } 6 \text { month-follow-up } \\
\text { Effect size (CAPS): Hedges' } g_{\text {post }}=-0.36^{\#} \text { (favoring active control } \\
\text { group) Hedges' } g_{3 \text { month }}=-0.47^{\#} \text { Hedges' } g_{6 \text { month }}=-0.62^{\#} \\
\text { Summary: Significant decrease on the CAPS for both treatment } \\
\text { groups. According to the authors there were no significant } \\
\text { differences between the groups }(p .70) \text {. Treatment gains were } \\
\text { maintained at } 6 \text { month follow up }\end{array}$ \\
\hline $\begin{array}{l}\text { Beidel et al. } \\
\text { (2017b) }\end{array}$ & USA & DSM-IV-TR & CAPS & $\begin{array}{l}\text { Pilot } \\
\text { study }\end{array}$ & $\begin{array}{l}\text { War veterans and } \\
\text { active duty } \\
\text { personnel with } \\
\text { past Iraq and } \\
\text { Afghanistan } \\
\text { deployment } \\
\text { Combat- related } \\
\text { PTSD symptoms }\end{array}$ & $\begin{array}{l}\text { Total participants: } N=112 \text {, drop-out: } \\
10(9.8 \%) \text {, mean age: } 37.1 \text {, gender: } \\
97(95 \%) \text { males }\end{array}$ & VRET with TMT & $\begin{array}{l}\text { Measurements: Pre, post, } 3 \text { and } 6 \text { month-follow-up } \\
\text { Effect size (CAPS): Cohen's } d_{\text {pre-post }}=2.06 \\
\text { Cohen's } d_{\text {pre-3month }}=\text { n.r. } \\
\text { Cohen's } d_{\text {pre-6month }}=\text { n.r. } \\
\text { Summary: Significant decrease on CAPS from pre to } \\
\text { post-treatment. Treatment gains were maintained at } 6 \text { month } \\
\text { follow up }\end{array}$ \\
\hline $\begin{array}{l}\text { Difede et al. } \\
\text { (2013) }\end{array}$ & USA & DSM-IV & CAPS & $\mathrm{RCT}$ & $\begin{array}{l}\text { Civilians, who had } \\
\text { PTSD symptoms } \\
\text { following exposure } \\
\text { to the WTC } \\
\text { attacks. }\end{array}$ & $\begin{array}{l}\text { Total participants: } N=25 \text {, drop-out: } \\
3(12 \%) \text {, mean age: } 45.7 \text {, gender: } 19 \\
(76 \%) \text { males } \\
\text { VR treatment group (VRET with DCS): } \\
n=13 \text {, drop-out: } 0(0 \%) \text {, mean age: } \\
47.7 \text {, gender: } 8 \text { ( } 61.5 \%) \text { males } \\
\text { Active control group (VRET with } \\
\text { placebo): } n=12 \text {, drop-out: } 3(25 \%) \text {, } \\
\text { mean age: } 43.7 \text {, gender: } 11 \\
(91.6 \%) \text { males }\end{array}$ & $\begin{array}{l}\text { VRET with DCS } \\
\text { vs. VRET with } \\
\text { placebo }\end{array}$ & $\begin{array}{l}\text { Measurements: Pre, post, and } 6 \text { month-follow-up } \\
\text { Effect size (CAPS): Hedges' } g_{\text {post }}=0.68 \text { (favoring VRET with } \\
\text { DCS) Hedges' } g_{6 m o n t h}=1.13 \\
\text { Summary: Significant decrease on the CAPS for both treatment } \\
\text { groups. At } 6 \text { month-follow-up VRET with DCS were superior to } \\
\text { active control group }\end{array}$ \\
\hline $\begin{array}{l}\text { Loucks et al. } \\
\text { (2018) }\end{array}$ & USA & DSM-5 & CAPS & $\begin{array}{l}\text { Pilot } \\
\text { study }\end{array}$ & $\begin{array}{l}\text { Military veterans } \\
\text { with PTSD } \\
\text { symptoms due } \\
\text { military sexual } \\
\text { trauma (MST) }\end{array}$ & $\begin{array}{l}\text { Total participants: } N=15 \text {, drop-out: } \\
6(40 \%) \text {, mean age: } 46 \text {, gender: } 11 \\
(73.4 \%) \text { females }\end{array}$ & VRET & $\begin{array}{l}\text { Measurements: Pre, post, and } 3 \text { month-follow-up } \\
\text { Effect size (CAPS): Cohen's } d_{\text {pre-post }}=1.11 \text { Cohen's } d_{\text {pre-3month }}= \\
\text { n.r. } \\
\text { Summary: This effect maintained at } 3 \text { month-follow-up. Results } \\
\text { suggest that VRET is a potential treatment for MST related PTSD }\end{array}$ \\
\hline
\end{tabular}




\begin{tabular}{|c|c|c|c|c|c|c|c|c|}
\hline References & Country & $\begin{array}{l}\text { Instrument } \\
\text { for PTSD } \\
\text { diagnosis }\end{array}$ & $\begin{array}{l}\text { Primary } \\
\text { outcome } \\
\text { variable }\end{array}$ & $\begin{array}{l}\text { Study } \\
\text { design }\end{array}$ & $\begin{array}{l}\text { Sample and } \\
\text { trauma type }\end{array}$ & Participants and drop-out & Intervention & Time points of measurements and main results \\
\hline $\begin{array}{l}\text { Maples-Keller } \\
\text { et al. (2018) }\end{array}$ & USA & DSM-5 & CAPS & $\mathrm{RCT}$ & $\begin{array}{l}\text { War veterans and } \\
\text { active duty } \\
\text { personnel with } \\
\text { past Iraq and } \\
\text { Afghanistan } \\
\text { deployment } \\
\text { Combat- related } \\
\text { PTSD symptoms }\end{array}$ & $\begin{array}{l}\text { Total participants: } N=27 \text {, drop-out: } \\
3(12 \%) \text {, mean age: } 35.4 \text {, gender: } 27 \\
(100 \%) \text { males } \\
\text { VR treatment group (VRET with } \\
\text { dexamethasone): } n=13 \text {, drop-out: } 0 \\
(0 \%) \text {, mean age: n.r., gender: } 13 \\
(100 \%) \text { males } \\
\text { Active control group (VRET with } \\
\text { placebo): } n=14 \text {, drop-out: } 3(25 \%) \text {, } \\
\text { mean age: n.r., gender: } 14 \\
(100 \%) \text { males }\end{array}$ & $\begin{array}{l}\text { VRET with } \\
\text { dexamethasone } \\
\text { vs. VRET with } \\
\text { placebo }\end{array}$ & $\begin{array}{l}\text { Measurements: Pre and post } \\
\text { Effect size (CAPS): Combined sample Cohen's } d_{\text {pre-post }}=\text { n.r. } \\
\text { Summary: Significant decrease on the CAPS for post-treatment } \\
\text { but no significant differences between groups }\end{array}$ \\
\hline $\begin{array}{l}\text { McLay et al. } \\
\text { (2017) }\end{array}$ & USA & DSM-IV & CAPS & $\mathrm{RCT}$ & $\begin{array}{l}\text { Active duty military } \\
\text { members with } \\
\text { past Iraq and } \\
\text { Afghanistan } \\
\text { deployment } \\
\text { Combat- related } \\
\text { PTSD symptoms }\end{array}$ & $\begin{array}{l}\text { Total participants: } N=81 \text {, drop-out: } \\
7(8 \%), \text { mean age: } 32.5 \text {, gender: } 78 \\
(96.3 \%) \text { males } \\
\text { VR treatment group (VRET with } \\
\text { immersive technology): } n=43, \\
\text { drop-out: } 7(16 \%) \text {, mean age: } 33 \text {, } \\
\text { gender: } 40 \text { (93\%) males } \\
\text { Active control group (VRET with } \\
\text { non-immersive technology): } n=38 \text {, } \\
\text { drop-out: } 0(0 \%), \text { mean age: } 32 \text {, } \\
\text { gender: } 38(100 \%) \text { males }\end{array}$ & $\begin{array}{l}\text { VRET with } \\
\text { immersive } \\
\text { technology vs. } \\
\text { VRET with } \\
\text { non-immersive } \\
\text { technology }\end{array}$ & $\begin{array}{l}\text { Measurements: Pre, post, and } 3 \text { month-follow-up } \\
\text { Effect size (CAPS): Hedges' } g_{\text {post }}=-0.33^{\#} \text { (favoring VRET with } \\
\text { non-immersive technology) Hedges' } g_{3 m o n t h}=0.15^{\#} \text { (favoring } \\
\text { VRET with immersive technology) } \\
\text { Summary: Significant decrease on the CAPS maintained over } 3 \\
\text { month-follow-up. No significant differences between groups } \\
\text { were found }\end{array}$ \\
\hline $\begin{array}{l}\text { Reger et al. } \\
\text { (2016) }\end{array}$ & USA & DSM-IV-TR & CAPS & RCT & $\begin{array}{l}\text { Active duty military } \\
\text { members with } \\
\text { past Iraq and } \\
\text { Afghanistan } \\
\text { deployment } \\
\text { Combat- related } \\
\text { PTSD symptoms }\end{array}$ & $\begin{array}{l}\text { Total participants: } N=162 \text {, drop-out: } \\
54(33 \%) \text {, mean age: } 30.2 \text {, gender: } \\
156(96.3 \%) \text { males } \\
\text { VR treatment group (VRET): } n=54 \text {, } \\
\text { drop-out: } 14 \text { (25\%), mean age: } 29.5 \text {, } \\
\text { gender: } 52 \text { (96\%) males } \\
\text { Active control group (prolonged } \\
\text { exposure): } n=54 \text {, drop-out: } 12 \\
\text { (22\%), mean age: } 30.8 \text {, gender: } 51 \\
\text { (94\%) males } \\
\text { Control group (waitlist): } n=54 \text {, } \\
\text { drop-out: } 7 \text { (12\%), mean age: } 30.3 \text {, } \\
\text { gender: } 53(98 \%) \text { males }\end{array}$ & $\begin{array}{l}\text { VRET vs. PE vs. } \\
\text { waiting list }\end{array}$ & $\begin{array}{l}\text { Measurements: Pre, post, } 3 \text { and } 6 \text { month-follow-up } \\
\text { Effect size (CAPS): VRET vs. PE: Hedges' } g_{\text {post }}=-0.38^{\#} \text { (favoring } \\
\text { PE) } \\
\text { VRET vs. Control: Hedges' } g_{\text {post }}=-0.39^{\#} \text { (favoring VRET) } \\
\text { PE vs. Control: Hedges' } g_{\text {post }}=0.84^{\# ~(f a v o r i n g ~ P E) ~} \\
\text { VRET vs. PE: Hedges' } 9_{3 m o n t h}=-0.63^{\# ~(f a v o r i n g ~ P E) ~} \\
\text { VRET vs. PE: Hedges' } g_{6 m o n t h}=-0.33^{\#} \text { (favoring PE) } \\
\text { Summary: According to the authors VRET and PE were superior } \\
\text { to wait-list at post-treatment. At } 3 \text { - and } 6 \text { month-follow-up VRET } \\
\text { were inferior to PE }\end{array}$ \\
\hline $\begin{array}{l}\text { Rothbaum } \\
\text { et al. (2014) }\end{array}$ & USA & DSM-IV-TR & CAPS & RCT & $\begin{array}{l}\text { War veterans with } \\
\text { Iraq and } \\
\text { Afghanistan } \\
\text { deployment } \\
\text { Combat- related } \\
\text { PTSD symptoms }\end{array}$ & $\begin{array}{l}\text { Total participants: } N=156 \text {, drop-out: } \\
59(37 \%) \text {, mean age: } 35.1 \text {, gender: } \\
148(94 \%) \text { males } \\
\text { VR treatment group (VRET with DCS): } \\
n=53 \text {, drop-out: } 25(47 \%) \text {, mean } \\
\text { age: } 34.9 \text {, gender: } 49(92 \%) \text { males } \\
\text { Active control group (VRET with } \\
\text { Alprazolam): } n=50 \text {, drop-out: } 15\end{array}$ & $\begin{array}{l}\text { VRET with DCS } \\
\text { vs. VRET with } \\
\text { Alprazolam vs. } \\
\text { VRET with } \\
\text { Placebo }\end{array}$ & $\begin{array}{l}\text { Measurements: Pre, post, 3, 6, and } 12 \text { month-follow-up } \\
\text { Effect size: n.r. and n.a.\# } \\
\text { Summary: All groups decreased significantly on the CAPS. The } \\
\text { effect maintained over } 12 \text { months-follow-up. At post-treatment } \\
\text { there was no significant difference between D-cycloserin and } \\
\text { placebo group on the CAPS. However, there was a significant } \\
\text { difference favoring placebo over alprazolam regarding the CAPS } \\
\text { at post-treatment }\end{array}$ \\
\hline
\end{tabular}


TABLE 1 | Continued

\begin{tabular}{|c|c|c|c|c|c|c|c|c|}
\hline References & Country & $\begin{array}{l}\text { Instrument } \\
\text { for PTSD } \\
\text { diagnosis }\end{array}$ & $\begin{array}{l}\text { Primary } \\
\text { outcome } \\
\text { variable }\end{array}$ & $\begin{array}{l}\text { Study } \\
\text { design }\end{array}$ & $\begin{array}{l}\text { Sample and } \\
\text { trauma type }\end{array}$ & Participants and drop-out & Intervention & Time points of measurements and main results \\
\hline & & & & & & $\begin{array}{l}\text { (30\%), mean age: } 36.2 \text {, gender: } 49 \\
\text { (98\%) males } \\
\text { Control group (VRET with placebo): } n \\
=53 \text {, drop-out: } 19(35 \%) \text {, mean age: } \\
\text { 34.3, gender: } 50(94 \%) \text { males }\end{array}$ & & \\
\hline $\begin{array}{l}\text { Van't Wout } \\
\text { et al. (2018) }\end{array}$ & USA & DSM-5 & PCL-5 & $\mathrm{RCT}$ & $\begin{array}{l}\text { War veterans with } \\
\text { Iraq and } \\
\text { Afghanistan } \\
\text { deployment } \\
\text { Combat-related } \\
\text { PTSD symptoms }\end{array}$ & $\begin{array}{l}\text { Total participants: } N=12 \text {, drop-out: } \\
\text { n.r., mean age: } 40.5 \text {, gender: } 12 \\
\text { (100\%) males } \\
\text { VR treatment group } \\
\text { (VRET with tDCS): } n=\text { n.r., drop-out: } \\
\text { n.r., mean age: } \text { n.r., gender: n.r. } \\
\text { Active control group (VRET with sham } \\
\text { tDCS): } n=\text { n.r., drop-out: n.r., mean } \\
\text { age: } \text {.r.r. } \\
\text { gender: } n \text {.r. }\end{array}$ & $\begin{array}{l}\text { VRET with tDCS } \\
\text { vs. VRET with } \\
\text { sham tDCS }\end{array}$ & $\begin{array}{l}\text { Measurements: Pre, post, and } 1 \text { month-follow-up } \\
\text { Effect size (PCL-5): Hedges' } g_{\text {post }}=0.20^{\#} \text { (favoring VRET with } \\
\text { tDCS) Cohen's } d_{1 \text { month }}=0.37 \\
\text { Summary: Both groups demonstrated significant reductions in } \\
\text { PCL scores. There were no significant differences between groups } \\
\text { at post time measurement, but VRET with tDCS were superior to } \\
\text { VRET sham tDCS at } 1 \text { month-follow-up }\end{array}$ \\
\hline $\begin{array}{l}\text { Bisson et al. } \\
(2020)\end{array}$ & $\begin{array}{l}\text { United Kingdom, } \\
\text { Wales }\end{array}$ & DSM-5 & CAPS-5 & $\mathrm{RCT}$ & $\begin{array}{l}\text { Military veterans } \\
\text { with treatment- } \\
\text { resistant and } \\
\text { combat-related } \\
\text { PTSD } \\
\text { Treatment- } \\
\text { resistance were } \\
\text { defined as } \\
\text { persisting PTSD } \\
\text { diagnosis following } \\
\text { a trauma- } \\
\text { focused therapy }\end{array}$ & $\begin{array}{l}\text { Total participants: } N=42 \text {, drop-out: } \\
6(14.2 \%), \text { mean age: } 42.1 \text {, gender: } \\
42(100 \%) \text { males } \\
\text { VR treatment group (3MDR): } n=21 \text {, } \\
\text { drop-out: } 4(19 \%), \text { mean age: } 40.2 \text {, } \\
\text { gender: } 21(100 \%) \text { males } \\
\text { Control group (waitlist): } n=21 \text {, } \\
\text { drop-out: } 2(4.7 \%), \text { mean age: } 44.0 \text {, } \\
\text { gender: } 21 \text { (100\%) males }\end{array}$ & $\begin{array}{l}\text { 3MDR vs. } \\
\text { waiting list } \\
\text { (participants } \\
\text { allocated to } \\
\text { waiting list } \\
\text { received } 3 \mathrm{MDR} \\
\text { after a delay of } \\
12 \text { weeks) }\end{array}$ & $\begin{array}{l}\text { Measurements: Pre, } 12 \text {, and } 26 \text { weeks after randomization } \\
\text { Effect size (CAPS-5): Cohen's } d_{12 \text { weeks }}=0.65 \text { (favoring } 3 \text { MDR) } \\
\text { Summary: Results indicated that } 3 \text { MDR reduces PTSD symptoms } \\
\text { in Veterans with treatment-resistant and combat-related PTSD. } \\
\text { The authors conclude that phase III trials with larger sample sizes } \\
\text { are warranted }\end{array}$ \\
\hline $\begin{array}{l}\text { Jetly et al. } \\
\text { (2017) }\end{array}$ & Canada & n.r. & PCL-5 & $\begin{array}{l}\text { Pilot } \\
\text { study }\end{array}$ & $\begin{array}{l}\text { Soldiers with } \\
\text { treatment-resistant } \\
\text { and combat- } \\
\text { related PTSD } \\
\text { Treatment- } \\
\text { resistant were } \\
\text { defined as patients } \\
\text { "who were non } \\
\text { responders to at } \\
\text { least one type of } \\
\text { trauma-focused. } \\
\text { psychotherapy" } \\
\text { (p. 1) }\end{array}$ & $\begin{array}{l}\text { Total participants: } N=8 \text {, drop-out: } 3 \\
(37.5 \%) \text { mean age: n.r., gender: n.r. }\end{array}$ & 3MDR & $\begin{array}{l}\text { Measurements: Pre and post } \\
\text { PCL-5 effect size (PCL-5): Cohen's } d_{\text {pre-post }}=\text { n.r. } \\
\text { Summary: Modest therapy gains for patients with } \\
\text { treatment-resistant and combat-related PTSD. The authors use } \\
\text { these preliminary results to conduct larger randomized controlled } \\
\text { trials (p. 2) }\end{array}$ \\
\hline
\end{tabular}


TABLE 1 | Continued

\begin{tabular}{|c|c|c|c|c|c|c|c|c|}
\hline References & Country & $\begin{array}{l}\text { Instrument } \\
\text { for PTSD } \\
\text { diagnosis }\end{array}$ & $\begin{array}{l}\text { Primary } \\
\text { outcome } \\
\text { variable }\end{array}$ & $\begin{array}{l}\text { Study } \\
\text { design }\end{array}$ & $\begin{array}{l}\text { Sample and } \\
\text { trauma type }\end{array}$ & Participants and drop-out & Intervention & Time points of measurements and main results \\
\hline $\begin{array}{l}\text { Nijdam and } \\
\text { Vermetten } \\
\text { (2018) }\end{array}$ & Netherlands & n.r. & n.r. & $\begin{array}{l}\text { Case } \\
\text { study }\end{array}$ & $\begin{array}{l}\text { War veteran with } \\
\text { Afghanistan } \\
\text { deployment } \\
\text { Treatment- } \\
\text { resistant and } \\
\text { combat- } \\
\text { related PTSD }\end{array}$ & $\begin{array}{l}\text { Participant: } N=1 \text {, drop-out: n.a., } \\
\text { age: } 51 \text {, gender: } 1(100 \%) \text { male }\end{array}$ & $3 M D R$ & $\begin{array}{l}\text { Measurements: n.r. } \\
\text { Effect size: n.a. } \\
\text { Summary: Positive treatment results for a single patient with } \\
\text { treatment-resistant and combat-related PTSD }\end{array}$ \\
\hline $\begin{array}{l}\text { Van Gelderen } \\
\text { et al. (2018) }\end{array}$ & Netherlands & n.r. & PCL-5 & $\begin{array}{l}\text { Case } \\
\text { study }\end{array}$ & $\begin{array}{l}\text { War veterans with } \\
\text { French foreign } \\
\text { legion, Lebanon } \\
\text { and Afghanistan } \\
\text { deployment } \\
\text { Treatment- } \\
\text { resistant and } \\
\text { combat- } \\
\text { related PTSD }\end{array}$ & $\begin{array}{l}\text { Total participants: } N=3 \text {, drop-out: } \\
\text { n.r., age: n.r., gender: } 3 \text { males }\end{array}$ & $3 M D R$ & $\begin{array}{l}\text { Measurements: } n . r . \\
\text { Effect size: n.a. } \\
\text { Summary: Positive treatment results for three patients with } \\
\text { treatment-resistant and combat-related PTSD. Before } 3 M D R \text { the } \\
\text { patients were treated with guidelines trauma focused interventions } \\
\text { for } 3 \text { months to } 6 \text { years without symptom relief }\end{array}$ \\
\hline $\begin{array}{l}\text { Van Gelderen } \\
\text { et al. (2020) }\end{array}$ & Netherlands & DSM-5 & CAPS-5 & $\mathrm{RCT}$ & $\begin{array}{l}\text { Veterans with } \\
\text { treatment-resistant } \\
\text { and } \\
\text { combat-related } \\
\text { PTSD } \\
\text { Treatment- } \\
\text { resistance were } \\
\text { defined as } \\
\text { persisting PTSD } \\
\text { diagnosis and lack } \\
\text { of improvement in } \\
\text { PTSD symptoms } \\
\text { following a } \\
\text { trauma-focused } \\
\text { therapy with a } \\
\text { treatment duration } \\
\text { of at least } \\
6 \text { months }\end{array}$ & $\begin{array}{l}\text { Total participants: } N=43 \text {, drop-out: } \\
3 \text { ( } 7 \%) \text {, mean age: } 42.1 \text {, gender: } 42 \\
(97 \%) \text { males } \\
\text { VR treatment group (3MDR): } n=22 \text {, } \\
\text { drop-out: } 2 \text { (9\%), mean age: } 42.4 \text {, } \\
\text { gender: } 21 \text { (95\%) males } \\
\text { Active control group (non-specific } \\
\text { treatment component control group, } \\
\text { NTCC): } n=21 \text {, drop-out: } 1 \text { (4.7\%), } \\
\text { mean age: } 41.9 \text {, gender: } 21 \\
\text { (100\%) males }\end{array}$ & $\begin{array}{l}\text { 3MDR (after } 6 \\
\text { sessions 3MDR } \\
\text { patients were } \\
\text { allowed to } \\
\text { receive other } \\
\text { treatments to } \\
\text { process any } \\
\text { therapeutic } \\
\text { material, mean } \\
\text { amount of } \\
\text { treatment weeks } \\
\text { =10.5) vs. } \\
\text { NTCC } \\
\text { (non-trauma- } \\
\text { focused } \\
\text { treatment e.g., } \\
\text { case } \\
\text { management, } \\
\text { stabilizing } \\
\text { interventions, } \\
\text { psychoeducation } \\
\text { etc. up to } 16 \\
\text { weeks; mean } \\
\text { amount of } \\
\text { treatment weeks } \\
=8.74 \text { ) }\end{array}$ & $\begin{array}{l}\text { Measurements: Pre, } 6,12 \text {, and } 16 \text { weeks after randomization } \\
\text { Effect size (CAPS-5): Hedges' } g_{6 \text { weeks }}=\text { n.r. and n.a. } \\
\text { Cohen's } d_{12 \text { weeks }}=\text { n.r. and n.a." } \\
\text { Cohen's } d_{16 \text { weeks }}=0.83 \text { (favoring 3MDR) } \\
\text { Summary: Results showed that 3MDR reduces PTSD symptoms } \\
\text { in Veterans with treatment-resistant and combat-related PTSD. } \\
\text { However, no significant differences were found for secondary } \\
\text { outcomes measures (e.g., PCL-5) and no long-term effects were } \\
\text { assessed. The authors conclude that phase III trials with larger } \\
\text { sample sizes are warranted }\end{array}$ \\
\hline
\end{tabular}




\begin{tabular}{|c|c|c|c|c|c|c|c|c|}
\hline References & Country & $\begin{array}{l}\text { Instrument } \\
\text { for PTSD } \\
\text { diagnosis }\end{array}$ & $\begin{array}{l}\text { Primary } \\
\text { outcome } \\
\text { variable }\end{array}$ & $\begin{array}{l}\text { Study } \\
\text { design }\end{array}$ & $\begin{array}{l}\text { Sample and } \\
\text { trauma type }\end{array}$ & Participants and drop-out & Intervention & Time points of measurements and main results \\
\hline $\begin{array}{l}\text { Vermetten } \\
\text { et al. (2013) }\end{array}$ & Netherlands & n.r. & PCL-5 & $\begin{array}{l}\text { Case } \\
\text { study }\end{array}$ & $\begin{array}{l}\text { War veterans with } \\
\text { treatment-resistant } \\
\text { and } \\
\text { combat-related } \\
\text { PTSD. }\end{array}$ & $\begin{array}{l}\text { Total participants: } N=2 \text {, drop-out: } \\
\text { n.r., age: n.r., gender: n.r. }\end{array}$ & $3 \mathrm{MDR}$ & $\begin{array}{l}\text { Measurements: n.r. } \\
\text { Effect size: n.a. } \\
\text { Summary: Preliminary results of two cases suggest that 3MDR is } \\
\text { perhaps a treatment for Treatment-resistant and } \\
\text { combat-related PTSD }\end{array}$ \\
\hline $\begin{array}{l}\text { Kengne et al. } \\
\text { (2018) }\end{array}$ & Canada & n.r. & n.r. & $\begin{array}{l}\text { Case } \\
\text { study }\end{array}$ & $\begin{array}{l}\text { Civilian truck } \\
\text { driver, who was } \\
\text { suffering from } \\
\text { PTSD following an } \\
\text { accident. }\end{array}$ & $\begin{array}{l}\text { Participant: } N=1 \text {, drop-out: n.r., } \\
\text { age: n.r., gender: } 1 \text { male }\end{array}$ & ACET & $\begin{array}{l}\text { Measurements: n.r. } \\
\text { Effect size: n.a. } \\
\text { Summary: PTSD related outcome measures were not reported. } \\
\text { However, the authors see this single case study as "the first step } \\
\text { for the validation" (p.8) of ACET and more studies are needed to } \\
\text { test the efficacy }\end{array}$ \\
\hline $\begin{array}{l}\text { Menelas et al. } \\
\text { (2018) }\end{array}$ & Canada & n.r. & PCL-5 & $\begin{array}{l}\text { Case } \\
\text { study }\end{array}$ & $\begin{array}{l}\text { Two civilian truck } \\
\text { drivers, who were } \\
\text { suffering from } \\
\text { PTSD following an } \\
\text { accident. }\end{array}$ & $\begin{array}{l}\text { Total participants: } N=2 \text {, drop-out: } \\
\text { n.r., age: } 50 \text { and } 36 \text { years old, } \\
\text { gender: } 2(100 \%) \text { males }\end{array}$ & ACET & $\begin{array}{l}\text { Measurements: n.r. } \\
\text { Effect size: n.r. } \\
\text { Summary: PCL-5 decreases were found for PTSD following a } \\
\text { truck accident. Authors highlighted that Patient b was "willing to } \\
\text { try a road test with a monitor" ( } p \text {. 11) after ACET was completed. } \\
\text { Further studies are needed to replicate and generalize } \\
\text { these results }\end{array}$ \\
\hline
\end{tabular}

3MDR, multi-modular motion-assisted memory desensitization and reconsolidation; ACET, action-centered exposure therapy; CAPS, clinician-administered PTSD scale, measured via CAPS total sum score; CAPS-5, clinician-administered PTSD scale for DSM-5, measured via CAPS-5 total sum score; DCS, D-cycloserine: DSM-5, Diagnostic and Statistical Manual of Mental Disorders 5th Edition; DSM-IV, Diagnostic and Statistical Manual of Mental Disorders 4th Edition; DSM-IV-TR, Diagnostic and Statistical Manual of Mental Disorders 4th Edition Text Revision; Hedges' $9^{\#}$, a Hedges'g marked by "\#” was recalculated. For the recalculation, we used means, standard deviations and sample sizes for the respective studies and followed the procedure by Hedges and OIkin (2014); min, minutes; MST, military sexual trauma; n.a., not applicable; n.a.", not applicable, because these studies did not reported standard deviations. Instead they reported mean values and 95\% confidence intervals; n.r., not reported; NTCC, non-specific treatment component control group; PCL-5, PTSD checklist for DSM-5, measured via PCL-5 total sum score; PE, prolonged exposure; post, post-treatment assessment; Pre, pre-treatment assessment; PTSD, post-traumatic stress disorder; RCT, randomized controlled trial; tDCS, transcranial direct current stimulation, patients in the tDCS group received additionally to VRET 25 minutes of two milliampere electrostimulation; TMT, trauma management therapy (Turner et al., 2005); VRET, virtual reality exposure therapy; WTC, World Trade Center. 
TABLE 2 | Results of the qualitative analysis for each study.

\begin{tabular}{|c|c|c|c|c|c|c|c|}
\hline \multirow[b]{2}{*}{ References } & \multicolumn{3}{|r|}{ Research question 1} & \multicolumn{2}{|c|}{ Research question 2} & \multirow{2}{*}{$\begin{array}{l}\begin{array}{l}\text { Research } \\
\text { question } 3\end{array} \\
\begin{array}{l}\text { Social and } \\
\text { spatial presence }\end{array}\end{array}$} & \multirow{2}{*}{$\begin{array}{l}\begin{array}{l}\text { Research } \\
\text { question } 4\end{array} \\
\text { Efficacy for } \\
\text { PTSD patients } \\
\text { with imaginary } \\
\text { problems }\end{array}$} \\
\hline & $\begin{array}{l}\text { Therapeutic } \\
\text { framework }\end{array}$ & Transfer to VR & Manualized & Hardware & Software & & \\
\hline
\end{tabular}

\section{Virtual reality exposure therapy (VRET)}

Arens (2014) PE Virtual re-creation of

the patient's traumatic $\quad$ - 29, 90 min therapy sessions over a period of 3 weeks Virtual recounting

$$
\text { exposure sessions: }
$$

- Nine exposure sessions with ca. 45-80 min of virtual exposure

- Virtual exposure was conducted until within session habituation

(50\% reduction of the SUD) was achieved

- Therefore, no specific time limit of virtual exposure was set

Medication:

- No

Homework:

- Yes, in vivo excersies were included

- Furthermore, if between session habituation (anxiety did not

increase during a virtual exposure session) was achieved, the

therapist switched to in vivo exposure

Virtual re-creation of

the patient's traumatic

Number of sessions and period of time:

recounting

- $29,90-120$ min therapy sessions over a period of 17 weeks Virtual

exposure sessions:

- 14 exposure sessions with ca. 20-100 min of virtual exposure

- Virtual exposure was conducted until within session habituation

( $50 \%$ reduction of the SUD) was achieved

- Therefore, no specific time limit of virtual exposure was set

Medication:

- No

Homework:

- Yes, in vivo excersies were included

- Furthermore, if between session habituation (anxiety did not

increase during a virtual exposure session) was achieved, the therapist switched to in vivo exposure

$\begin{aligned} & \text { Beidel et al. PE } \\ & \text { (2017b) }\end{aligned}$
$\begin{aligned} & \text { Virtual re-creation of } \\ & \text { the patient's traumatic }\end{aligned}$

Number of sessions and period of time:

the patient's traumatic - 29, 90-120 min therapy sessions over a period of

recounting

three weeks Virtual exposure sessions:
HMD

(eMaginZ800) Iraq/Afghanistan

HMD (HMZ-T3W) BRAVEMIND

Was not measured Was not evaluated

- 14 exposure sessions with ca. 20-100 min of virtual exposure

- Virtual exposure was conducted until within session habituation

( $50 \%$ reduction of the SUD) was achieved

- Therefore, no specific time limit of virtual exposure was set

Medication:

- No

Homework:

- Yes, in vivo excersies were included 
TABLE 2 | Continued

Research question 1

Research question 2

Research

question 3

Research

Manualized

Hardware

Software

Social and

question 4

References

Therapeutic
framework

Transfer to VR

spatial presen

Efficacy for

PTSD patients

with imaginary

problems

- Furthermore, if between session habituation (anxiety did not

increase during a virtual exposure session) was achieved, the

therapist switched to in vivo exposure

$\begin{array}{ll}\begin{array}{l}\text { Difede et al. PE } \\ \text { (2013) }\end{array} & \begin{array}{l}\text { Virtual re-creation of } \\ \text { the patient's traumatic } \\ \text { recounting }\end{array}\end{array}$

- Ten exposure sessions with ca. 45 min of virtual exposure
Number of sessions and period of time: exposure sessions:

- Referring to Difede et al. (2007) the virtual exposure was also conducted until within-session habituation was attained (50\% reduction of SUD)

Medication:

- Yes, patients took D-cycloserin (100 mg) or placebo 90 min before the exposure session

Homework:

- Yes, in vivo excersies were included

Virtual re-creation of the Number of sessions and period of time:

context and settings $\quad$ - Six to 12, 90 min therapy sessions over a period of 6 to

factors. For MST it is 9 weeks Virtual exposure sessions:

not attempt to recreate - Four to 10 exposure sessions with ca. 45 min of virtual exposure

a sexual assault.

- Did n.r. whether within-session habituation in sense of $50 \%$

reduction of SUD was attained

Medication:

- No

Homework:

- Yes, in vivo excersies were included

Number of sessions and period of time:

Virtual re-creation of

the patient's traumatic

- Seven to twelve, 90 min therapy sessions

12 weeks Virtual exposure sessions:

- Six to 11 exposure sessions with ca. 30-45 min of virtual exposure

- Did n.r. whether within-session habituation in sense of 50\%

reduction of SUD was attained

Medication:

- Yes, patients took dexamethasone $(0.5 \mathrm{mg})$ or placebo the night before virtual exposure

Homework:

- No
HMD

(eMaginZ800)

BRAVEMIND

Was not measured Was not evaluated

Virtual version of the WTC attack

Was not measured Was not evaluated

$\begin{array}{ll}\text { HMD } & \text { Virtual } \\ (\text { eMaginZ800) } & \text { Iraq/Afghanistan }\end{array}$

Was not measured Was not evaluated 
TABLE 2 | Continued

\begin{tabular}{|c|c|c|c|c|c|c|c|}
\hline \multirow[b]{2}{*}{ References } & \multicolumn{3}{|r|}{ Research question 1} & \multicolumn{2}{|c|}{ Research question 2} & \multirow{2}{*}{$\begin{array}{l}\begin{array}{l}\text { Research } \\
\text { question } 3\end{array} \\
\text { Social and } \\
\text { spatial presence }\end{array}$} & \multirow{2}{*}{$\begin{array}{l}\begin{array}{l}\text { Research } \\
\text { question } 4\end{array} \\
\text { Efficacy for } \\
\text { PTSD patients } \\
\text { with imaginary } \\
\text { problems }\end{array}$} \\
\hline & $\begin{array}{l}\text { Therapeutic } \\
\text { framework }\end{array}$ & Transfer to VR & Manualized & Hardware & Software & & \\
\hline $\begin{array}{l}\text { McLay et al. } \\
\text { (2017) }\end{array}$ & PE & $\begin{array}{l}\text { Virtual re-creation of } \\
\text { the patient's traumatic } \\
\text { recounting }\end{array}$ & $\begin{array}{l}\text { Number of sessions and period of time: } \\
\text { - Eight to } 12,90 \text { min therapy sessions over a period of } \\
9 \text { weeks Virtual exposure sessions: } \\
\text { - Five to nine exposure sessions with } 30-45 \text { min of virtual exposure } \\
\text { - Did n.r. whether within-session habituation in sense of } 50 \% \\
\text { reduction of SUD was attained } \\
\text { Medication: } \\
\text { - No } \\
\text { Homework: } \\
\text { - Yes, in vivo excersies were included }\end{array}$ & $\begin{array}{l}\text { HMD } \\
\text { (eMaginZ800) }\end{array}$ & $\begin{array}{l}\text { Virtual } \\
\text { Iraq/Afghanistan }\end{array}$ & Was not measured & Was not evaluated \\
\hline $\begin{array}{l}\text { Reger et al. } \\
\text { (2016) }\end{array}$ & PE & $\begin{array}{l}\text { Virtual re-creation of } \\
\text { the patient's traumatic } \\
\text { recounting }\end{array}$ & $\begin{array}{l}\text { Number of sessions and period of time: } \\
\text { - Ten, } 90-120 \text { min therapy sessions over a period of } 5 \text { to } \\
10 \text { weeks Virtual exposure sessions: } \\
\text { - Eight exposure sessions with ca. } 45 \text { min of virtual exposure } \\
\text { - Did n.r. whether within-session habituation in sense of } 50 \% \\
\text { reduction of SUD was attained } \\
\text { Medication: } \\
\text { - No } \\
\text { Homework: } \\
\text { - Yes, in vivo excersies were included }\end{array}$ & $\begin{array}{l}\text { HMD } \\
\text { (eMaginZ800) }\end{array}$ & $\begin{array}{l}\text { Virtual } \\
\text { Iraq/Afghanistan }\end{array}$ & Was not measured & Was not evaluated \\
\hline $\begin{array}{l}\text { Rothbaum } \\
\text { et al. (2014) }\end{array}$ & PE & $\begin{array}{l}\text { Virtual re-creation of } \\
\text { the patient's traumatic } \\
\text { recounting }\end{array}$ & $\begin{array}{l}\text { Number of sessions and period of time: } \\
\text { - Six, } 90 \text { min therapy sessions over a period of } 6 \text { weeks } \\
\text { Virtual exposure sessions: } \\
\text { - Five exposure sessions with } 45 \text { min of virtual exposure } \\
\text { Medication: } \\
\text { - Yes, patients took D-cycloserine }(50 \mathrm{mg}) \text {, alprazolam }(0.25 \mathrm{mg}) \text { or } \\
\text { the placebo medication } 30 \text { min before exposure } \\
\text { - Did n.r. whether within-session habituation in sense of } 50 \% \\
\text { reduction of SUD was attained } \\
\text { Homework: } \\
\text { - No }\end{array}$ & $\begin{array}{l}\text { HMD } \\
\text { (eMaginZ800) }\end{array}$ & $\begin{array}{l}\text { Virtual } \\
\text { Iraq/Afghanistan }\end{array}$ & Was not measured & Was not evaluated \\
\hline
\end{tabular}


TABLE 2 | Continued

\begin{tabular}{|c|c|c|c|c|c|c|c|}
\hline \multirow[b]{2}{*}{ References } & \multicolumn{3}{|r|}{ Research question 1} & \multicolumn{2}{|c|}{ Research question 2} & \multirow{2}{*}{$\begin{array}{l}\begin{array}{l}\text { Research } \\
\text { question } 3\end{array} \\
\begin{array}{l}\text { Social and } \\
\text { spatial presence }\end{array}\end{array}$} & \multirow{2}{*}{$\begin{array}{l}\text { Research } \\
\text { question } 4 \\
\text { Efficacy for } \\
\text { PTSD patients } \\
\text { with imaginary } \\
\text { problems }\end{array}$} \\
\hline & $\begin{array}{l}\text { Therapeutic } \\
\text { framework }\end{array}$ & Transfer to VR & Manualized & Hardware & Software & & \\
\hline $\begin{array}{l}\text { Van't Wout } \\
\text { et al. (2018) }\end{array}$ & PE & $\begin{array}{l}\text { Virtual re-creation of } \\
\text { the patient's traumatic } \\
\text { recounting }\end{array}$ & $\begin{array}{l}\text { Number of sessions and period of time: } \\
\text { - Six, } 90 \text { min therapy sessions over a period of } 2 \text { weeks } \\
\text { - Virtual exposure sessions: } \\
\text { - Six exposure sessions with } 30-45 \text { min of virtual exposure } \\
\text { Medication: } \\
\text { - Yes, during virtual exposure patients received tDCS or sham tDCS } \\
\text { - Did n.r. whether within-session habituation in sense of } 50 \% \\
\text { reduction of SUD was attained } \\
\text { Homework: } \\
\text { - No }\end{array}$ & $\begin{array}{l}\text { HMD } \\
\text { (eMaginZ800) }\end{array}$ & $\begin{array}{l}\text { Virtual } \\
\text { Iraq/Afghanistan }\end{array}$ & Was not measured & Was not evaluated \\
\hline
\end{tabular}

\section{Multi-modular motion-assisted memory desensitization and reconsolidation (3MDR)}

\begin{tabular}{|c|c|c|c|c|c|c|c|}
\hline $\begin{array}{l}\text { Bisson et al. } \\
(2020)\end{array}$ & EMDR & $\begin{array}{l}\text { Patients walk toward } \\
\text { individualized } \\
\text { trauma-related } \\
\text { symbolic images in a } \\
\text { CAVE }\end{array}$ & $\begin{array}{l}\text { Number of sessions and period of time: } \\
\text { - Nine, } 60 \text { min therapy sessions over a period of } 9 \text { weeks Virtual } \\
\text { - } \text { Six virtual exposure sessions with ca. } 45 \text { min of virtual exposure } \\
\text { - Did n.r. whether within-session habituation in sense of } 50 \% \\
\text { reduction of SUD was attained } \\
\text { Medication: } \\
\text { - No } \\
\text { Homework: } \\
\text { - No }\end{array}$ & CAVE (GRAIL) & $\begin{array}{l}\text { Individualized } \\
\text { trauma-associated } \\
\text { images }\end{array}$ & Was not measured & $\begin{array}{l}\text { Was not } \\
\text { evaluated. } \\
\text { However, only } \\
\text { treatment-resistant } \\
\text { PTSD patients } \\
\text { were included in } \\
\text { this study }\end{array}$ \\
\hline $\begin{array}{l}\text { Jetly et al. } \\
\text { (2017) }\end{array}$ & EMDR & $\begin{array}{l}\text { Patients walk toward } \\
\text { individualized } \\
\text { trauma-related } \\
\text { symbolic images in a } \\
\text { CAVE }\end{array}$ & $\begin{array}{l}\text { Number of sessions and period of time: } \\
\text { - Nine, ca. } 40 \text { min therapy sessions over a period of } 8 \text { weeks } \\
\text { - Virtual exposure sessions: } \\
\text { - Six virtual exposure sessions with ca. } 30 \text { min of virtual exposure } \\
\text { - Did n.r. whether within-session habituation in sense of } 50 \% \\
\text { reduction of SUD was attained } \\
\text { Medication: } \\
\text { - No } \\
\text { Homework: } \\
\text { - No }\end{array}$ & CAVE (CAREN) & $\begin{array}{l}\text { Individualized } \\
\text { trauma-associated } \\
\text { images }\end{array}$ & Was not measured & $\begin{array}{l}\text { Was not } \\
\text { evaluated. } \\
\text { However, only } \\
\text { treatment-resistant } \\
\text { PTSD patients } \\
\text { were included in } \\
\text { this study }\end{array}$ \\
\hline
\end{tabular}


TABLE 2 | Continued

\begin{tabular}{|c|c|c|c|c|c|c|c|}
\hline \multirow[b]{2}{*}{ References } & \multicolumn{3}{|r|}{ Research question 1} & \multicolumn{2}{|c|}{ Research question 2} & \multirow{2}{*}{$\begin{array}{l}\begin{array}{l}\text { Research } \\
\text { question } 3\end{array} \\
\begin{array}{l}\text { Social and } \\
\text { spatial presence }\end{array}\end{array}$} & \multirow{2}{*}{$\begin{array}{l}\text { Research } \\
\text { question } 4 \\
\text { Efficacy for } \\
\text { PTSD patients } \\
\text { with imaginary } \\
\text { problems }\end{array}$} \\
\hline & $\begin{array}{l}\text { Therapeutic } \\
\text { framework }\end{array}$ & Transfer to VR & Manualized & Hardware & Software & & \\
\hline $\begin{array}{l}\text { Nijdam and } \\
\text { Vermetten } \\
\text { (2018) }\end{array}$ & EMDR & $\begin{array}{l}\text { Patients walk toward } \\
\text { individualized } \\
\text { trauma-related } \\
\text { symbolic images in a } \\
\text { CAVE }\end{array}$ & $\begin{array}{l}\text { Number of sessions and period of time: } \\
\text { - Was not reported Virtual exposure sessions: } \\
\text { - Six virtual exposure sessions (duration of virtual exposure was not } \\
\text { reported) } \\
\text { - Did n.r. whether within-session habituation in sense of } 50 \% \\
\text { reduction of SUD was attained } \\
\text { Medication: } \\
\text { - No } \\
\text { Homework: } \\
\text { - No }\end{array}$ & CAVE (CAREN) & $\begin{array}{l}\text { Individualized } \\
\text { trauma-associated } \\
\text { images }\end{array}$ & Was not measured & $\begin{array}{l}\text { Was not } \\
\text { evaluated. } \\
\text { However, only } \\
\text { treatment-resistant } \\
\text { PTSD patients } \\
\text { were included in } \\
\text { this study }\end{array}$ \\
\hline $\begin{array}{l}\text { Van Gelderen } \\
\text { et al. (2018) }\end{array}$ & EMDR & $\begin{array}{l}\text { Patients walk toward } \\
\text { individualized } \\
\text { trauma-related } \\
\text { symbolic images in a } \\
\text { CAVE }\end{array}$ & $\begin{array}{l}\text { Number of sessions and period of time: } \\
\text { - Was not reported Virtual exposure sessions: } \\
\text { - Was not reported } \\
\text { - Did n.r. whether within-session habituation in sense of 50\% } \\
\text { reduction of SUD was attained } \\
\text { Medication: } \\
\text { - No } \\
\text { Homework: } \\
\text { - No }\end{array}$ & CAVE (CAREN) & $\begin{array}{l}\text { Individualized } \\
\text { trauma-associated } \\
\text { images }\end{array}$ & Was not measured & $\begin{array}{l}\text { Was not } \\
\text { evaluated. } \\
\text { However, only } \\
\text { treatment-resistant } \\
\text { PTSD patients } \\
\text { were included in } \\
\text { this study }\end{array}$ \\
\hline $\begin{array}{l}\text { Van Gelderen } \\
\text { et al. (2020) }\end{array}$ & EMDR & $\begin{array}{l}\text { Patients walk toward } \\
\text { individualized } \\
\text { trauma-related } \\
\text { symbolic images in a } \\
\text { CAVE }\end{array}$ & $\begin{array}{l}\text { Number of sessions and period of time: } \\
\text { - Six }+10 \text { optional, } 70-90 \text { min therapy session over a period of } \\
6-16 \text { weeks Virtual exposure sessions: } \\
\text { - Six virtual exposure sessions with } 30-45 \text { min of virtual exposure } \\
\text { - Did n.r. whether within-session habituation in sense of } 50 \% \\
\text { reduction of SUD was attained } \\
\text { Medication: } \\
\text { - No } \\
\text { Homework: } \\
\text { - No }\end{array}$ & CAVE (CAREN) & $\begin{array}{l}\text { Individualized } \\
\text { trauma-associated } \\
\text { images }\end{array}$ & Was not measured & $\begin{array}{l}\text { Was not } \\
\text { evaluated. } \\
\text { However, only } \\
\text { treatment-resistant } \\
\text { PTSD patients } \\
\text { were included in } \\
\text { this study }\end{array}$ \\
\hline
\end{tabular}


TABLE 2 | Continued

\begin{tabular}{|c|c|c|c|c|c|c|c|}
\hline \multirow[b]{2}{*}{ References } & \multicolumn{3}{|r|}{ Research question 1} & \multicolumn{2}{|c|}{ Research question 2} & \multirow{2}{*}{$\begin{array}{l}\begin{array}{l}\text { Research } \\
\text { question } 3\end{array} \\
\begin{array}{l}\text { Social and } \\
\text { spatial presence }\end{array}\end{array}$} & \multirow{2}{*}{$\begin{array}{l}\text { Research } \\
\text { question } 4 \\
\text { Efficacy for } \\
\text { PTSD patients } \\
\text { with imaginary } \\
\text { problems }\end{array}$} \\
\hline & $\begin{array}{l}\text { Therapeutic } \\
\text { framework }\end{array}$ & Transfer to VR & Manualized & Hardware & Software & & \\
\hline $\begin{array}{l}\text { Vermetten } \\
\text { et al. (2013) }\end{array}$ & EMDR & $\begin{array}{l}\text { Patients walk toward } \\
\text { individualized } \\
\text { trauma-related } \\
\text { symbolic images in a } \\
\text { CAVE }\end{array}$ & $\begin{array}{l}\text { Number of sessions and period of time: } \\
\text { - Six, } 45 \text { min therapy session over a period of } 4 \text { weeks Virtual } \\
\text { exposure sessions: } \\
\text { - Four virtual exposure sessions (duration of virtual exposure was n.r.) } \\
\text { - Did n.r. whether within-session habituation in sense of } 50 \% \\
\text { reduction of SUD was attained } \\
\text { Medication: } \\
\text { - No } \\
\text { Homework: } \\
\text { - No }\end{array}$ & CAVE (CAREN) & $\begin{array}{l}\text { Individualized } \\
\text { trauma-associated } \\
\text { images }\end{array}$ & Was not measured & $\begin{array}{l}\text { Was not } \\
\text { evaluated. } \\
\text { However, only } \\
\text { treatment-resistant } \\
\text { PTSD patients } \\
\text { were included in } \\
\text { this study }\end{array}$ \\
\hline
\end{tabular}

\section{Action-centered exposure therapy (ACET)}

\section{Kengne et al. Inhibitory Active interaction with a Number of sessions and period of time:}

(2018) learning virtual

trauma-associated environment
- Eight therapy session (session duration and period of weeks

were n.r.) Virtual exposure sessions:

- Six virtual exposure sessions (duration of virtual exposure was n.r.)

- Did n.r. whether within-session habituation in sense of $50 \%$

reduction of SUD was attained

Medication:

- No

Homework:

- No
HMD (HMZ-T2) Self-programmed Was not measured Was not evaluated virtual country

roads, highways,

and cities
HMD (HMZ-T2) Self-programmed Was not measured Was not evaluated virtual country roads, highways, and cities
- Eight therapy session over a period of 4 weeks

- Six vitual exposure sessions (duration of virtual exposure was not

- Did n.r. whether within-session habituation in sense of 50\%

reduction of SUD was attained

Medication:

- No

Homework:

- No

3MDR, multi-modular motion-assisted memory desensitization and reconsolidation; ACET, action-centered exposure therapy; CAREN, computer-assisted rehabilitation environment; CAVE, cave automatic virtual environment; EMDR, eye movement desensitization and reprocessing; GRAIL, gait real-time analysis interactive lab; HMD, head-mounted display; mg, milligram; min, minute; MST, military sexual trauma; n.r., not reported; PE, prolonged exposure; PTSD, post-traumatic stress disorder; SUD, subjective units of distress; tDCS, transcranial direct current stimulation; VR, virtual reality; VRET, virtual reality exposure therapy; WTC, World Trade Center. 
TABLE 3 | Overview of results.

\section{General overview}

Included studies

Country

7 RCTs (patients: $N=555$ )

2 Pilot studies (patients: $N=127$ )

1 Case study (patient: $N=1$ )

- USA: 10/10 studies

Trauma type

\section{Research questions}

1.1) Therapeutic framework ( $\boldsymbol{\Lambda}$

1.2) How it was transferred to VR (summary) ( +

\section{3) Manualized ( $\star$ )}

1.3.1) Number of sessions and period of time

1.3.2) Virtual exposure sessions

\subsection{3) Medication}

1.3.4) Homework (in vivo exercise)

2.1) Hardware (*)
8/10 war veterans with combat-related PTSD

- 1/10 war veterans with PTSD after MST

- 1/10 civilians with PTSD after a terrorist attack (WTC)

$\lambda P E$

+ Summary: Virtual re-creation of the patient's traumatic recounting during virtual exposure

^ 7/10 studies used six to 12, ca. 90 min therapy sessions over a period of 6 to 12 weeks

*3/10 studies used 29, 90-120 min therapy sessions over a period of 3-17 weeks

$\star 7 / 10$ studies used six to 11 virtual exposure sessions with ca. 30-45 min of virtual exposure

\ $3 / 10$ studies used nine to 14 virtual exposure sessions with ca. 20-100 min of virtual exposure

$\star 4 / 10$ studies explicitly reported that virtual exposure was conducted until within-session habituation was attained

* 4/10 studies used medication (Alp., DCS, DMT, tDCS)

$\star 7 / 10$ studies included in vivo exercises between the therapy sessions (e.g., visiting crowded places, sitting with one's back to a doorway)

* 8/10 studies used eMaginZ800 (HMD, released in 2005)

* 1/10 study used ProView XL-50 (HMD, released in 2006)

* 1/10 study used HMZ-T3W (HMD, released in 2013)

2 RCTs (patients: $N=85$ )

- 1 Pilot study (patients: $N=8$ )

- 3 Case studies (patients: $N=6$ )

- Canada: 1/6 study

- Netherlands: 4/6 studies

- UK: $1 / 6$ study

- 6/6 war veterans with combat-related and treatment-resistant PTSD

ᄉ EMDR

+ Summary: Patients walk toward individualized trauma-related symbolic images, which increased simultaneously and continuously in size. After each image patients perform virtual dual-attention tasks to stimulate the patient's working memory and facilitate reconsolidation

$\star 1 / 6$ used six, 45 min therapy sessions over a period of 4 weeks

^ 2/6 studies used nine, 40-60 min therapy sessions over a period of 8 to 9 weeks

$\star 1 / 6$ used nine plus 10 optional $70-90$ min therapy sessions over a period of 6-16 weeks

$\star 2 / 6$ did n.r. the number and time of therapy sessions

$\star 5 / 6$ studies used six virtual exposure sessions, with ca. 30-45 min of virtual exposure

$\star 1 / 6$ n.r. the number and time of virtual exposure sessions

$\star 0 / 6$ studies did n.r. whether virtual exposure was conducted until within-session habituation was attained

$\star 6 / 6$ did not use medication

^ 6/6 studies did not include in vivo exercise

* 5/6 studies used CAREN (computer-assisted rehabilitation environment, first release in 2000, assignable to a CAVE)

- 1/6 study used GRAll (Gait Real-time Analysis Interactive Lab, assignable to a CAVE)
- 2 Case studies (patients: $N=2$ )

- Canada: 2/2 studies

- 2/2 civilian truck drivers with PTSD after a truck driver accident

A Inhibitory learning

+ Summary: Active interaction with a virtual trauma-associated environment during virtua exposure

$\star 2 / 2$ used eight therapy sessions

$\star 1 / 2$ reported a period of 4 weeks and the other one did n.r. the period of weeks

$\star$ No study reported the exact time of therapy sessions

$\star 2 / 2$ studies used six virtual exposure sessions

$\star$ The duration of virtual exposure was n.r.

$\star 0 / 2$ studies did n.r. whether virtual exposure was conducted until within-session habituation was attained

$\star 2 / 2$ did not use medication

$\star 2 / 2$ studies did not include in vivo exercise

* HMZ-T2 (HMD, released in 2012) * Logitech G27 3 Driving Force GTracing wheel (wheel, gas, and brake pedal) 


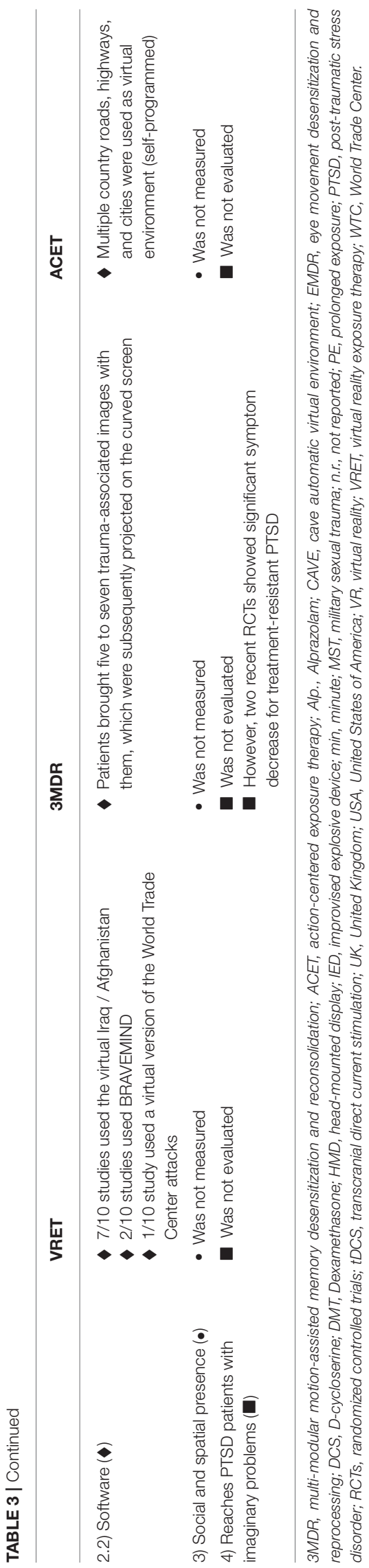

was used. Difede et al. (2013) applied a ProView XL-50 HMD (released in 2006), which has a pixel resolution of $1,042 \times 768$ with a $40^{\circ}$ horizontal field of view. Beidel et al. (2017a) applied an HMZ-T3W HMD (released in 2013), which has a pixel resolution of $1,280 \times 720$ with a $45^{\circ}$ horizontal field of view.

Most of the studies (90\%) used the virtual Iraq/Afghanistan developed by Rizzo et al. (2005) or the updated version Bravemind (Rizzo et al., 2017) as a virtual trauma environment. Bravemind is based on the first version of Virtual Iraq/Afghanistan (Rizzo et al., 2005) and was updated and further developed in 2011 using the Unity $3 d$ game engine (Rizzo and Shilling, 2017; Rizzo et al., 2017). The four original scenarios were rebuilt using the Unity $3 \mathrm{~d}$ engine, and 10 additional preprogrammed situations were added (e.g., a rural Afghan village and a roadway checkpoint). In both virtual environments, the patients control their perspectives through head movements and navigate through the virtual environment using a gaming controller.

Difede et al. (2013) used a virtual version of the World Trade Center (WTC) attack as a virtual environment that consists of 13 preprogrammed scenarios. Based on the idea of a hierarchical exposure, these 13 preprogrammed scenarios vary in the intensity of the presented material. The first scene starts with a jet flying over the WTC towers without a crash, hit, or explosion. The following scenario shows a jet crashing into the first tower, but the tower does not collapse. The last scene shows the jets crash into both towers. The towers collapse, dust clouds appear, and human screaming can be heard.

\section{Spatial and Social Presence in VRET}

The patients' spatial or social presence during virtual trauma exposure was not measured in any of the 10 studies.

\section{VRET for PTSD Patients With Imaginary Problems}

Whether VRET is particularly effective for patients with PTSD and imagination difficulties has not been examined in any of the aforementioned studies.

\section{Additional Information (Study Objectives and Efficacy)}

A total of 10 (55.5\%) VRET studies were included in the present scoping review, with seven being RCTs (Difede et al., 2013; Rothbaum et al., 2014; Reger et al., 2016; Beidel et al., 2017a; McLay et al., 2017; Maples-Keller et al., 2018; Van't Wout et al., 2018), two pilots (Beidel et al., 2017b; Loucks et al., 2018) and one single-case study (Arens, 2014).

The studies' objectives were quite heterogeneous. Three out of 10 studies explored whether the efficacy of VRET may be increased through additional medication (Difede et al., 2013; Rothbaum et al., 2014; Maples-Keller et al., 2018). MaplesKeller et al. (2018) examined whether the administration of dexamethasone improved the efficacy of VRET compared to placebo treatment. Rothbaum et al. (2014) analyzed to what extent D-cycloserine and alprazolam influenced the efficacy of VRET, also in comparison to a placebo group. Furthermore, Difede et al. (2013) examined whether D-cycloserine improved the efficacy of VRET in comparison to a placebo group. None 
of these studies revealed a significant difference at the post time measurement compared to the placebo group (Difede et al., 2013; Rothbaum et al., 2014; Maples-Keller et al., 2018). All studies used the Clinician-Administered PTSD Scale (CAPS; Blake et al., 1995) as the primary outcome measure.

Further three out of 10 studies examined whether VRET may be integrated into Trauma Management Therapy (TMT; Turner et al., 2005). Three studies with different designs showed that TMT in combination with VRET led to a clinically relevant and significant reduction of PTSD symptoms from pre- to post and 6-month-follow-up (Arens, 2014; Beidel et al., 2017a,b). Beidel et al. (2017a) compared whether the combination of TMT with VRET is more effective than psychoeducation modules with VRET. They did not reveal any significant differences between the conditions at post assessment and at the 6-month-follow-up (Beidel et al., 2017a).

Two feasibility studies examined whether VRET can be used to treat MST (Loucks et al., 2018) and whether transcranial direct current stimulation (tDCS; Nitsche et al., 2008) increases the efficacy of VRET (Van't Wout et al., 2018). These studies showed that VRET reduced the PTSD symptoms after MST significantly from pre- to post and 3-month-follow-up (Loucks et al., 2018), and that tDCS could improve the efficacy of VRET (Van't Wout et al., 2018).

Reger et al. (2016) conducted an RCT to examine whether there was a significant difference between the efficacy of VRET, $\mathrm{PE}$ and a minimal attention waitlist. They showed that VRET as well as PE were superior to the control group (Reger et al., 2016). Contrary to expectation, there was no significant difference between VRET and PE at post-treatment, whereas VRET was significantly inferior to PE after a 3-month-follow-up (Reger et al., 2016).

McLay et al. (2017) also conducted an RCT to examine whether the efficacy differed between immersive (HMD) and non-immersive (PC) VRET. McLay et al. (2017) found no significant difference in CAPS scores between the conditions at the post-treatment and 3-month-follow-up.

\section{Multi-Modular Motion-Assisted Memory Desensitization and Reconsolidation (3MDR) \\ Therapeutic Framework Transferred Into 3MDR}

The 3MDR framework is a model for the treatment of PTSD, focusing on therapy-resistant PTSD (trPTSD; Koek et al., 2016), where the mechanisms of EMDR, multi-sensory input, walking on a treadmill, and dual attention tasks are combined (Vermetten et al., 2013; Van Gelderen et al., 2018, 2020). Van Gelderen et al. (2018) hypothesized that avoidance behavior of trPTSD is particularly pronounced, making access to traumatic memories more difficult. Therefore, new therapeutic approaches are needed to reduce avoidance behavior, increase therapy adherence, and reduce symptoms of trPTSD (Vermetten et al., 2013; Nijdam and Vermetten, 2018; Van Gelderen et al., 2018, 2020). All studies included war veterans with combat-related and treatmentresistant PTSD.
In addition, all studies used the same 3MDR manualized treatment protocol, which posits 6 weekly 90 min sessions (Van Gelderen et al., 2020). Each session includes a preliminary briefing (20 min), a virtual trauma confrontation on a treadmill (for approximately $50 \mathrm{~min}$ ), and a review discussion $(20 \mathrm{~min}$ ). Before the virtual exposure, one or two preparatory sessions are conducted ( $45 \mathrm{~min}$ ). Here, the goal is to inform the patient about the procedure, offer psychoeducation, and select photographs and music that will be integrated into the virtual trauma confrontation (Van Gelderen et al., 2020).

One central part of the preparatory sessions is the selection process of 10-20 highly affective photographs associated with the traumatic experience (Bisson et al., 2020; Van Gelderen et al., 2020). The patients can choose any photograph they want to see (Bisson et al., 2020). For instance, they can choose their own photographs or select images from the Internet (e.g., landscapes or an area on Google Maps) (Van Gelderen et al., 2020). The selected images are then arranged according to the distress they evoke. Therefore, each photograph will be rated with a SUD using a score of $0-10$. For each session, a maximum of seven pictures were selected and arranged according to their SUD scores (Bisson et al., 2020). The chosen images will be projected on the CAVE and enable the creation of an idiosyncrasy virtual environment rather than a generic one (Van Gelderen et al., 2020). The virtual confrontation will start with the least emotionally image (with the lowest SUD) and will finish with the highest. This procedure can be repeated throughout the sessions, and it is also possible to reorder or reselect the images for each session (Van Gelderen et al., 2020).

As another precondition, the patient selects two different pieces of music (e.g., songs or natural sounds) (Bisson et al., 2020). The first is for a warm-up and the second is for a cooldown walk. The warm-up music aims to evoke associations related to the trauma-related period and to bring the patient back to the time of the trauma (Bisson et al., 2020; Van Gelderen et al., 2020). The cool-down music helps the patient return to the here and now and should not be trauma related.

After the preparatory sessions and preliminary discussion, the virtual trauma confrontation starts with a technical briefing (Van Gelderen et al., 2020). Therefore, the operator, who is in the room for the entire duration of the virtual exposure, tethers the patient in the safety harness, provides information regarding safety, and adjusts the speed of the treadmill. The introductory phase then begins, which consists of the warm-up music and an initial walk until the patient reaches the first virtual tunnel (Bisson et al., 2020; Van Gelderen et al., 2020).

The patients enter the first tunnel and by doing so move toward the first selected image, which increases simultaneously and continuously in size (Van Gelderen et al., 2020). While moving forward, the patient should describe the photograph and the associative memories. The patients should then talk about their feelings evoked by the image at that moment (now feelings). When the patients verbalize their current feelings, the therapist repeats them, the operator types them in, and they appear in real-time on the screen (Van Gelderen et al., 2020).

After this reprocessing of trauma-related memories and feelings, the reconsolidation process starts with a dual-attention 
task (Van Gelderen et al., 2018). Therefore, a red ball appears on the screen. This red ball moves from left to right with different numbers. The patients' task is to call out these numbers and simultaneously concentrate on the memories and feelings they just named on the screen. The patients should call out six or more numbers. If they are unable to do so, the task is extended by the operator (Van Gelderen et al., 2020).

This sequence will be repeated until the patient completes the last (7th) image (Bisson et al., 2020). The outro sequence then begins. The patients' cool-down music plays, and the treadmill begins to slow down and finally stops. The operator helps the patient out of the harness, and the virtual trauma confrontation ends with a brief discussion between the patient, operator, and therapist (Van Gelderen et al., 2020).

\section{Hardware and Software Used for 3MDR}

In five out six studies, the computer-assisted rehabilitation environment (CAREN) was used as an immersive technology. CAREN, released in 2000, consists of a projection surface 6-m long and curved $180^{\circ}$, with a 5.1 Dolby Surround audio system, a tracking system, and an omnidirectional treadmill (Mert et al., 2013; Vermetten et al., 2013; Jetly et al., 2017; Van Gelderen et al., 2018, 2020). Bisson et al. (2020) used the gait real-time analysis interactive lab (GRAIL), which consists of a $180^{\circ}$ projection screen with four projectors, a surround and motion-capture system, and an instrumented dual-belt treadmill (Bisson et al., 2020).

In all studies the patients brought their own trauma-associated images with them, which were subsequently projected on the curved screen (Vermetten et al., 2013; Jetly et al., 2017; Nijdam and Vermetten, 2018; Van Gelderen et al., 2018, 2020; Bisson et al., 2020). These 2D images were used as the virtual environment in all 3MDR studies.

\section{Spatial and Social Presence in 3MDR}

The patients' spatial or social presence was not measured in any of the six studies.

\section{MDR for PTSD Patients With Imaginary Problems}

Whether 3MDR is particularly effective for patients with PTSD and imagination difficulties was not evaluated in any of the studies. However, all 3MDR studies were conducted with a focus on veterans or soldiers with treatment-resistant PTSD. Treatment resistance was defined as a persisting PTSD diagnosis following trauma-focused therapy. Van Gelderen et al. (2020) reported that the patients had, on average, four unsuccessful PTSD treatments. The sample by Bisson et al. (2020) also consisted of patients who had tried at least one trauma-focused psychological treatment (EMDR or trauma-focused cognitive behavioral therapy).

\section{Additional Information (Study Objectives and Efficacy)}

A total of six (33.3\%) 3MDR studies were included in the present scoping review, with two being RCTs (Bisson et al., 2020; Van Gelderen et al., 2020), one pilot (Jetly et al., 2017), and three single-case studies (Vermetten et al., 2013; Nijdam and Vermetten, 2018; Van Gelderen et al., 2018). The objectives of the studies were considerably homogenous. Whether 3MDR is an efficient treatment tool for treatment-resistant PTSD was examined in all the $3 \mathrm{MDR}$ studies.

Bisson et al. (2020) conducted an RCT of British military servicemen with trPTSD to examine whether $3 \mathrm{MDR}$ was superior to the waitlist group. Patients in the waitlist group received 3MDR after a delay of 6 weeks (Bisson et al., 2020). As expected, $3 \mathrm{MDR}$ was significantly superior to the waitlist at post-treatment $(d=0.65)$, and there was no significant difference between the groups after 26 weeks (at this time, both groups received 3MDR) (Bisson et al., 2020).

Van Gelderen et al. (2020) also conducted an RCT of Dutch veterans with trPTSD to examine whether $3 \mathrm{MDR}$ is superior to an active control group. Patients in the active control group received non-trauma-focused treatments such as stabilizing intervention or cognitive behavioral therapy without exposure or cognitive restructuring of trauma-related cognitions for up to 16 weeks. Patients in the $3 \mathrm{MDR}$ group received six standardized $3 \mathrm{MDR}$ sessions. In addition, there was the possibility of 10 optional sessions, depending on patients' need or the indications of the therapists. As expected, $3 \mathrm{MDR}$ was significantly superior to the active control group after 16 weeks of treatment $(d=0.83)$. However, immediately after the six 3MDR sessions (after week 6), no significant differences were shown.

The other pilot and single-case studies also revealed clinically relevant symptom reductions for trPTSD (Vermetten et al., 2013; Jetly et al., 2017; Nijdam and Vermetten, 2018; Van Gelderen et al., 2018).

\section{Action-Centered Exposure Therapy (ACET) Therapeutic Framework Transferred Into ACET}

In ACET, the focus is on patients' active interaction with the virtual trauma-associated environment. Thus, the approach of inhibitory learning in exposure therapy is used. This is the main difference between ACET and VRET, which utilizes the emotional processing theory by creating virtual visualizations to supplement the patients' traumatic recounting (Foa and Kozak, 1986; Foa and McNally, 1996; Rizzo and Shilling, 2017; Kengne et al., 2018; Menelas et al., 2018). It is assumed that these virtual visualizations activate the pathological fear structure more controllably (Leaman et al., 2013). However, VRET does not focus on active interactions between the patients and virtual trauma-related environment; rather, it supplements the patients' imagination. In contrast, ACET focuses on the active interaction with the virtual trauma-associated environment, with the aim of reducing PTSD symptoms by altering the anxiety structure through new secondary inhibitory learning (Craske et al., 2014; Kengne et al., 2018; Menelas et al., 2018). In ACET, patients are encouraged to gain new experiences, for example, by accomplishing missions such as driving a truck from an industrial area to a rural one (Kengne et al., 2018). To accomplish these missions, the patients must interact with the virtual traumarelated environment (e.g., in addition to navigating and changing perspectives using head movements, it is intended that the patients explore the virtual environment independently; they can select and manipulate virtual objects and interact with 
artificial-intelligence-programmed road drivers) (Kengne et al., 2018; Menelas et al., 2018). Habituation is not emphasized here; rather, the focus is on the new secondary inhibitory learning, which is derived from the active interactions within the virtual environment (Craske et al., 2014; Kengne et al., 2018).

Both single-case studies were conducted with a focus on PTSD related to truck driver accidents and used the same treatment protocol, which consisted of eight sessions (Kengne et al., 2018; Menelas et al., 2018). The first two sessions were preparatory sessions. Here, the patient should become familiar with the virtual environment, learn how to interact with it, and reconnect with driving mechanisms without direct exposure to a truck (Menelas et al., 2018). Therefore, the authors programmed a flying carpet, which was controlled using a steering wheel, gas, and brake pedal, as applied in the later sessions using a truck (Kengne et al., 2018; Menelas et al., 2018). The author chose this allegory to allow the patients to reconnect with the driving mechanism without direct exposure (Kengne et al., 2018; Menelas et al., 2018).

In the third session, the patients were exposed to a truck (Kengne et al., 2018). They walked around a virtual parking lot and could choose their favorite one. In the following session they could customize the truck, for example, change the color or seat materials. In sessions four and five, the patients drove the truck, with several missions to accomplish. For instance, they were required to drive the truck to a specific destination (Kengne et al., 2018; Menelas et al., 2018). By doing so, they were exposed to different weather conditions (sun, rain, and snow) and different roads (e.g., small streets without traffic or highways with high traffic). In session six, the patients were indirectly exposed to trauma by passing a burning truck on the roadside. After this high-level exposure, sessions seven and eight focused on the driving itself with similar content as provided in sessions four and five (Kengne et al., 2018; Menelas et al., 2018).

\section{Hardware and Software Used for ACET}

Both single-case studies used the HMZ-T2, an HMD by Sony (released in 2012) and the head-tracking system Natural Point TrackIR 5 (Kengne et al., 2018; Menelas et al., 2018). Based on the Game Engine Unreal Engine 4, multiple country roads, highways, and cities with different weather (sun, snow, rain, etc.) and daylight conditions were programmed. Using a steering wheel, gas, and brake pedal (Logitech G27 3 Driving Force GTracing wheel), the patient steered the truck through the virtual environment as close to reality as possible (Kengne et al., 2018; Menelas et al., 2018). Furthermore, it was possible to additionally add car traffic programmed with artificial intelligence. The authors ensured that this simulated road traffic was as close to reality as possible to allow the patient to independently collect new and corrective experiences.

\section{Spatial and Social Presence in ACET}

The spatial or social presence was not measured in any of the single-case studies (Kengne et al., 2018; Menelas et al., 2018).

\section{ACET for PTSD Patients With Imaginary Problems}

Whether ACET is particularly effective for PTSD patients with imagination difficulties was not evaluated in any of the abovementioned studies (Kengne et al., 2018; Menelas et al., 2018).

\section{Additional Information (Study Objectives and Efficacy)}

The present scoping review identified two (11.1\%) case studies on ACET (Kengne et al., 2018; Menelas et al., 2018). Both studies showed initial evidence that ACET can reduce PTSD symptoms related to truck driving accidents. The authors highlighted that, after ACET, one patient ( patient $_{\mathrm{b}}$ ) was able to complete the driving training and is now working again as a truck driver (Menelas et al., 2018). However, larger replication studies are required to confirm the efficacy of ACET. Therefore, the results must be interpreted with caution (Kengne et al., 2018; Menelas et al., 2018).

\section{DISCUSSION}

The present scoping review revealed that, at present, research on the subject encompasses three different approaches for virtual trauma interventions, which are based on three different traumafocused therapeutic approaches. Regarding the first research question (1), the results showed that VRET is based on PE, 3MDR is based on EMDR, and ACET is based on an inhibitory learning model. In accordance with previous reviews, this review also found that the empirical focus of previous research has been on VRET (10 out of 18 studies, with 7 out of 9 RCTs), whereas 3 MDR ( 6 out of 18, with 2 out of 9 RCTs) and ACET ( 2 out of 18) represent developments that have not been considered to a large extent in previous research. However, during the next few years, it is likely that the focus of the examination will shift to $3 \mathrm{MDR}$. Currently, one such study is registered in the International Standard Randomized Controlled Trial Number (ISRCTN) registry (ISRCTN11264368). Another study is registered at clinicaltrials.gov (NCT03796936), and yet another study is registered in the Netherlands Trial Register (NL6837). In contrast only a single pre-registration for VRET was found at clinicaltrials.gov (NCT0135263) and none was found for ACET 4 . Based on the three pre-registrations for $3 \mathrm{MDR}$ and the results of the two recent published RCTs (Bisson et al., 2020; Van Gelderen et al., 2020), we likely assume that the research focus will shift to $3 \mathrm{MDR}$.

Furthermore, to answer the second part of the first research question (1) our results showed that the method of transferring the therapeutic framework into VR varies for the three types of virtual trauma interventions (see Table 3). VRET focuses on supplementing patients' traumatic recounting through virtual visualizations, which activate the pathological fear structure controllably and in conformity with the emotional processing theory (Foa and McNally, 1996; McLean and Foa, 2011). Subsequently, the affective memory can be modified through

${ }^{4}$ Supplementary Figures 1-3 contains the flow diagram on the pre-registration search. 
habituation and extinction experience. This process can change the pathological fear structure and reduce PTSD symptoms. For this purpose, pre-programmed virtual scenarios, which can be partly modified to aid the patient's traumatic recounting, were used (Leaman et al., 2013; Rizzo and Shilling, 2017). In $3 \mathrm{MDR}$, the patients walk toward individualized trauma-related symbolic images in a CAVE, which increase continuously in size, aiming to break and minimize avoidance patterns (Van Gelderen et al., 2020). At the end of each image presentation, the patient performs a virtual dual-attention task to stimulate their working memory and facilitate the reconsolidation of the traumatic memory trace. In ACET, the focus is on the active interactions between the patients and trauma-related virtual environment. The patients are expected to gain new corrective experiences by independently accomplishing missions in the trauma-related virtual environment by actively interacting with it (e.g., exploring and manipulating the virtual environment) (Kengne et al., 2018).

For the third part of the first research question (1) the results demonstrated that the manualization of the treatment protocols also varies according to the virtual trauma confrontations. Furthermore, the results showed that the VRET protocols are heterogeneous. In contrast, the virtual exposure treatment protocols used in all the 3MDR and ACET studies were the same. Most of the studies on VRET (70\%) included 6-11 virtual exposure sessions, lasting 30-45 min. In the remaining studies, the virtual exposure sessions were conducted until the withinsession habituation was attained. Therefore, the virtual exposure sessions at the beginning were longer (ca. $100 \mathrm{~min}$ ) than the later ones (ca. $20 \mathrm{~min}$ ). All the studies were based on the emotional processing theory; therefore, habituation was a prerequisite for treatment success. However, the difference in exposure duration raises the question of whether the predetermined time window in the $70 \%$ of the VRET studies was sufficiently long for the patients to experience habituation after the fear structure activation. Therefore, future studies should examine whether the duration of virtual exposure moderates the efficacy of VRET. Furthermore, the treatment protocols of VRET varied according to whether medication or at-home in vivo exposure exercises were included. This heterogeneity made the comparability of the VRET studies challenging. It is unclear whether the efficacy of VRET is attributable to the virtual visualization of the patient's recounting or other factors (e.g., exposure duration, medication, and at-home in vivo exposure exercises). In contrast, in the $3 \mathrm{MDR}$ studies, the same treatment protocols were used in the virtual exposure sessions, which were six, weekly sessions of $90 \mathrm{~min}$. The procedure of the virtual exposure sessions was also standardized. Although the study designs of the two recent 3MDR RCTs were different (Bisson et al., 2020; Van Gelderen et al., 2020), the virtual trauma exposure was identical. Future systematic reviews can build on this and examine the efficacy of the virtual exposure sessions. In case of ACET, the same treatment protocol was used in both the single-case studies, which included two preparatory sessions and six indirect exposure sessions (Kengne et al., 2018; Menelas et al., 2018). These sessions consisted of several missions for the patients to accomplish. It was assumed that the new inhibitory experiences during the indirect exposure sessions would reduce the PTSD symptoms. However, there are only two single-case studies from the same authors. Therefore, it is necessary to interpret the results with caution.

Referring to the first part of the second research question (2), the results showed that HMDs were used in 12 studies, and CAVEs were applied in six. Detailed analyses revealed that $75 \%$ of the HMDs were released in 2005-2006, and the remaining 25\% were released in 2012-2013. Therefore, the effects of technological advances on the efficacy or acceptance of virtual trauma interventions are currently unknown.

Regarding the second part of the second research question (2), the results showed that the software used in VRET, 3MDR, and ACET are also different. Pre-programmed virtual scenarios are used in VRET. The patients control their perspectives through head movements and navigate through the virtual environment using a gaming controller. Further active interactions, such as selecting and manipulating virtual objects, independently exploring the virtual environment, or interacting with avatars, are not intended. Therefore, it is unclear how this lack of interaction influences the sense of spatial or social presence (Sherman and Craig, 2019). Moreover, because the scenarios are preprogrammed, it is not possible to create a virtual traumarelated environment that completely matches the patient's recounting, thereby leading to incongruency. Therefore, breaks in the sense of spatial or social presence and plausibility may occur (Slater, 2009; Oh et al., 2019). In contrast, a completely unique virtual environment is used in $3 \mathrm{MDR}$. For this purpose, individualized trauma-associated, fear-inducing, and avoided images related to the patient's traumatic experience are projected onto a CAVE. In this CAVE, the patient approaches the images, which simultaneously increase in size. However, in contrast to VRET, these are 2D representations without stereoscopy. As Cummings and Bailenson (2015) demonstrated in their meta-analysis, there is a medium positive correlation between stereoscopy and spatial presence. Similar to VRET, ACET uses computer-programmed virtual environments. However, it focuses on the active interaction between the patient and virtual environment. In addition to navigating and changing perspectives using head movements, it is intended that the patients explore the virtual environment independently; they can select and manipulate virtual objects and interact with artificialintelligence-programmed road drivers. ACET integrates several factors to enhance the possibility of patients experiencing spatial and social presence.

Overall, the choice of hardware and software depends on the type of virtual trauma intervention. The advantages and disadvantages mentioned earlier can influence the sense of spatial and social presence, which is presumed to be a major influence on treatment success in all virtual trauma interventions. These differences are also relevant from a cost-efficiency perspective. As mentioned in the introduction, VRET and ACET have the advantage of low hardware costs. However, programming a virtual trauma-related environment is expensive and timeconsuming. Additionally, a virtual trauma-related environment is always customized to one specific trauma type and population, limiting its generalizability. Table 1 supports this hypothesis, 
showing that the subjects of almost all VRET studies (90\%) were US veterans with combat-related PTSD. In contrast, there are almost no software costs in 3MDR because it uses traumarelated images. However, the hardware costs for a CAVE are still high. Nevertheless, once a CAVE has been installed, 3MDR is more likely to be generalizable to different types of traumas and samples because there are almost no software costs.

Regarding the third research question (3), the results revealed that neither the spatial nor social presence was assessed in any of the 18 studies; thus, no information could be retrieved regarding the potential effect of spatial or social presence on the efficacy of virtual trauma interventions. This is a surprising result as all virtual trauma interventions (VRET, 3MDR, and ACET) are based on the assumption that the sense of presence is an important prerequisite. Therefore, it is not comprehensible why none of the selected studies has empirically examined it. This illustrates the need for future research to examine whether spatial presence constitutes a crucial mechanism in shaping the efficacy of virtual trauma interventions, as previously assumed by some researchers (Kothgassner et al., 2019).

Regarding the fourth research question (4), it was equally surprising that there is no empirical evidence on whether virtual trauma intervention is particularly effective for PTSD patients with imagination difficulties. For example, for VRET, it is assumed that virtual visualizations of the patient's recounting can particularly help patients who are unable or unwilling to imagine the traumatic event. However, this assumption cannot be verified because there is no empirical evidence of the usefulness of virtual trauma intervention for such patients. Therefore, future research is required to establish whether virtual trauma interventions are particularly effective for PTSD patients with imagination difficulties.

However, recent RCTs support the argument that virtual trauma interventions can reach patients who cannot benefit from established treatment guidelines (Bisson et al., 2020; Van Gelderen et al., 2020). The reason trPTSD patients benefit from 3MDR has not been examined, and the results should be interpreted with caution because they are not sufficiently confirmed, owing to small sample sizes. However, these results demonstrate that $3 \mathrm{MDR}$ can make clinically meaningful difference to the symptoms of trPTSD patients who do not benefit from imaginal trauma-focused guideline procedures. Based on these results, clinicians can weigh the acquisition costs of a CAVE against the long-term therapy costs for trPTSD patients. This could justify the acquisition costs for a CAVE and enhance the obtainability of $3 \mathrm{MDR}$ in future. It should be noted that such a cost-efficiency decision is currently possible only for 3MDR because there is no empirical evidence of the efficacy of VRET and ACET for trPTSD.

\section{Limitations}

The findings of the present scoping review must be viewed critically based on various limitations. For example, the search was only conducted in English and German so we might have missed important studies written in other languages. In line with this, we found that the included studies were mostly conducted in English-speaking countries (55.5\% in North
America, $16.6 \%$ in Canada, 5.5\% in the United Kingdom) and the remaining studies were conducted in the Netherlands. In order to counteract a potential country bias, pre-registration databases of various countries (Korea, China, Brazil, Cuba, Iran, Germany, Netherlands, EU clinical trials register, ClinicalTrials.gov and ISCRCTN registry) were screened to ascertain whether other countries are planning to or are currently examining virtual trauma interventions. A total of four pre-registrations were identified, one in the Netherlands, two in North America and one in Canada. These findings and the study characteristics of the 18 included studies suggest that there is a geographic pattern for virtual trauma interventions, which includes predominantly English-speaking countries and the Netherlands. However, given that our search is based on English and German keywords, a language bias cannot be ruled out so the findings should be interpreted with caution.

In addition we would like to point out that even though VRET was the most frequently published therapeutic virtual intervention for PTSD patients, and thus may be regarded a pioneer in the field, to date there are only results from the US, specifically from American war veterans. Also, the $3 \mathrm{MDR}$ studies focused on war veterans. Only the case studies on ACET examined PTSD following truck driver accidents. Hence, less is known about the efficacy of virtual trauma intervention for non-deployment related PTSD (e.g., domestic violence, natural disasters). Finally, patients in the included studies were predominantly male adults. Therefore, future research is needed to replicate the results with different populations (e.g., female, children, adolescents, and elderly adults) and with a broader range of traumatic events to confirm the generalizability of the findings.

\section{CONCLUSION}

Previous studies have focused on the efficacy of VRET, which is one type of virtual trauma intervention. Therefore, this scoping review examined other virtual trauma interventions. We identified three different virtual trauma intervention approaches, namely, VRET, 3MDR, and ACET. These three approaches are based on different in sensu therapeutic frameworks. They vary in terms of transferring them into VR and of the treatment protocols. Although the procedures used for the virtual exposure in 3MDR and ACET are the same, we observed heterogeneity in VRET; therefore, we identified potentially confounding variables (exposure duration, medication, and at-home in vivo exposure) for the efficacy of VRET. Furthermore, our results demonstrated that the hardware and software are determined by the specific virtual trauma approach. From a cost-efficiency perspective, 3MDR seems promising. Although it has high hardware costs, two recent 3MDR RCTs demonstrated that it is efficient for trPTSD. Based on these results, clinicians can weigh the acquisition costs of a CAVE against the longterm therapy costs for trPTSD patients. Such a cost-efficiency decision currently applies only to 3MDRs because we found no empirical evidence of the efficacy of VRET and ACET for trPTSD. 
We revealed other research gaps. Although all the studies posited that the sense of presence was an important prerequisite for treatment success, it was not examined in any of the studies included in this review. Therefore, we strongly recommend that future studies examine whether the sense of presence (spatial and/or social) influences treatment success. Moreover, it also remains unclear to what extent more recent technological advances relating to immersive technology have affected virtual trauma interventions because most of the HMDs utilized were released over 15 years ago. Furthermore, we did not identify any empirical evidence that virtual exposure is particularly helpful for patients with imaginations difficulties. This is surprising because it is a frequently cited argument for VRET. Thus, future research should examine whether virtual exposure can benefit PTSD patients with imagination difficulties. Finally, our descriptive results revealed that most studies examined male soldiers with combat-related PTSD. Therefore, replication studies with different trauma types and populations are warranted.

\section{DATA AVAILABILITY STATEMENT}

The original contributions presented in the study are included in the article/supplementary materials, further inquiries can be directed to the corresponding author/s.

\section{REFERENCES}

Acarturk, C., Konuk, E., Cetinkaya, M., Senay, I., Sijbrandij, M., Gulen, B., et al. (2016). The efficacy of eye movement desensitization and reprocessing for post-traumatic stress disorder and depression among Syrian refugees: results of a randomized controlled trial. Psychol. Med. 46, 2583-2593. doi: $10.1017 /$ S0033291716001070

Acierno, R., Knapp, R., Tuerk, P., Gilmore, A. K., Lejuez, C., Ruggiero, K., et al. (2016). A non-inferiority trial of prolonged exposure for posttraumatic stress disorder: in person versus home-based telehealth. Behav. Res. Ther. 89, 57-65. doi: 10.1016/j.brat.2016.11.009

Arens, A. M. (2014). Trauma management therapy for a veteran with cooccurring combat PTSD and hallucinations. Clin. Case Stud. 14, 115-128. doi: $10.1177 / 1534650114541324$

Asukai, N., Saito, A., Tsuruta, N., Kishimoto, J., and Nishikawa, T. (2010). Efficacy of exposure therapy for Japanese patients with posttraumatic stress disorder due to mixed traumatic events: a randomized controlled study. J. Trauma. Stress 23, 744-750. doi: 10.1002/jts.20589

Beidel, D. C., Frueh, B. C., Neer, S. M., Bowers, C. A., Trachik, B., Uhde, T. W., et al. (2017a). Trauma management therapy with virtual-reality augmented exposure therapy for combat-related PTSD: a randomized controlled trial. J. Anxiety Disord. 61, 64-74. doi: 10.1016/j.janxdis.2017. 08.005

Beidel, D. C., Frueh, B. C., Neer, S. M., and Lejuez, C. W. (2017b). The efficacy of trauma management therapy: a controlled pilot investigation of a three-week intensive outpatient program for combat-related PTSD. J. Anxiety Disord. 50, 23-32. doi: 10.1016/j.janxdis.2017.05.001

Biocca, F., Harms, C., and Burgoon, J. K. (2003). Toward a more robust theory and measure of social presence: review and suggested criteria. Presence 12, 456-480. doi: 10.1162/105474603322761270

Bisson, J. I., van Deursen, R., Hannigan, B., Kitchiner, N., Barawi, K., Jones, K., et al. (2020). Randomized controlled trial of multi-modular motionassisted memory desensitization and reconsolidation (3MDR) for male military veterans with treatment-resistant post-traumatic stress disorder. Acta Psychiatr. Scand. 142, 141-151. doi: 10.1111/acps.13200

\section{AUTHOR CONTRIBUTIONS}

TK, R-JG, HH, and HS designed the review. TK conducted the systematic searches and completed data extraction. TK and R-JG wrote the first draft of the manuscript. AF, OK, $\mathrm{HH}$, and HS contributed extensively to the first draft and to the final manuscript. All authors read and approved the final manuscript prior to submission.

\section{FUNDING}

Open access funding was provided by University Hospital Hamburg-Eppendorf (UKE).

\section{ACKNOWLEDGMENTS}

We thank Vivien Tahal (VT) and Philipp Skiberowski (PS) for their help during the screening process and Medina Nehls for correcting the English manuscript.

\section{SUPPLEMENTARY MATERIAL}

The Supplementary Material for this article can be found online at: https://www.frontiersin.org/articles/10.3389/fpsyg. 2020.562506/full\#supplementary-material

Blake, D. D., Weathers, F. W., Nagy, L. M., Kaloupek, D. G., Gusman, F. D., Charney, D. S., et al. (1995). The development of a clinician-administered PTSD scale. J. Trauma. Stress 8, 75-90. doi: 10.1002/jts.2490080106

Borrego, A., Latorre, J., Lloréns, R., Noé, E., and Keshner, E. A. (2015). "Low-cost, room-size, and highly immersive virtual reality system for virtual and mixed reality applications," in 2015 International Conference on Virtual Rehabilitation (ICVR) (Valencia: IEEE), 273-277.

Botella, C., Fernández-Álvarez, J., Guillén, V., García-Palacios, A., and Baños, R. (2017). Recent progress in virtual reality exposure therapy for phobias: a systematic review. Curr. Psychiatry Rep. 19:42. doi: 10.1007/s11920-017-0788-4

Botella, C., Serrano, B., Baños, R. M., and Garcia-Palacios, A. (2015). Virtual reality exposure-based therapy for the treatment of post-traumatic stress disorder: a review of its efficacy, the adequacy of the treatment protocol, and its acceptability. Neuropsychiatr. Dis. Treat. 11, 2533-2545. doi: 10.2147/NDT.S89542

Capezzani, L., Ostacoli, L., Cavallo, M., Carletto, S., Fernandez, I., Solomon, R., et al. (2013). EMDR and CBT for cancer patients. Comparative study of effects on PTSD, anxiety, and depression. J. EMDR Prac. Res. 7, 134-143. doi: 10.1891/1933-3196.7.3.134

Carl, E., Stein, A. T., Levihn-Coon, A., Pogue, J. R., Rothbaum, B., Emmelkamp, P., et al. (2018). Virtual reality exposure therapy for anxiety and related disorders: a meta-analysis of randomized controlled trials. J. Anxiety Disord. 61, 27-36. doi: 10.1016/j.janxdis.2018.08.003

Carletto, S., Borghi, M., Bertino, G., Oliva, F., Cavallo, M., Hofmann, A., et al. (2016). Treating post-traumatic stress disorder in patients with multiple sclerosis: a randomized controlled trial comparing the efficacy of eye movement desensitization and reprocessing and relaxation therapy. Front. Psychol. 7:526. doi: 10.3389/fpsyg.2016.00526

Carlson, J. G., Chemtob, C. M., Rusnak, K., Hedlund, N. L., and Muraoka, M. Y. (1998). Eye movement desensitization and reprocessing (EDMR) treatment for combat-related posttraumatic stress disorder. J. Trauma. Stress 11, 3-24. doi: 10.1023/A:1024448814268

Cipresso, P., Giglioli, I. A. C., Raya, M. A., and Riva, G. (2018). The past, present, and future of virtual and augmented reality research: a network and cluster analysis of the literature. Front. Psychol. 9:2086. doi: 10.3389/fpsyg.2018.02086 
Coburn, J. Q., Freeman, I., and Salmon, J. L. (2017). A review of the capabilities of current low-cost virtual reality technology and its potential to enhance the design process. J. Comput. Inform. Sci. Eng. 17, 60-61. doi: 10.1115/1. 4036921

Craske, M. G., Treanor, M., Conway, C. C., Zbozinek, T., and Vervliet, B. (2014). Maximizing exposure therapy: an inhibitory learning approach. Behav. Res. Ther. 58, 10-23. doi: 10.1016/j.brat.2014.04.006

Cukor, J., Gerardi, M., Alley, S., Reist, C., Roy, M., Rothbaum, B. O., et al. (2015). "Virtual reality exposure therapy for combat-related PTSD," in Posttraumatic Stress Disorder and Related Diseases in Combat Veterans, ed E. C. Ritchie (Cham: Springer International Publishing), 69-83.

Cummings, J. J., and Bailenson, J. N. (2015). How immersive is enough? A metaanalysis of the effect of immersive technology on user presence. Media Psychol. 19, 272-309. doi: 10.1080/15213269.2015.1015740

Deng, W., Hu, D., Xu, S., Liu, X., Zhao, J., Chen, Q., et al. (2019). The efficacy of virtual reality exposure therapy for PTSD symptoms: a systematic review and meta-analysis. J. Affect. Disord. 257, 698-709. doi: 10.1016/j.jad.2019.07.086

Devilly, G. J., and Spence, S. H. (1999). The relative efficacy and treatment distress of EMDR and a cognitive-behavior trauma treatment protocol in the amelioration of posttraumatic stress disorder. J. Anxiety Disord. 13, 131-157. doi: 10.1016/S0887-6185(98)00044-9

Devilly, G. J., Spence, S. H., and Rapee, R. M. (1998). Statistical and reliable change with eye movement desensitization and reprocessing. Treating trauma within a veteran population. Behav. Ther. 29, 435-455. doi: 10.1016/S0005-7894(98)80042-7

Difede, J., Cukor, J., Jayasinghe, N., Patt, I., Jedel, S., Spielman, L., et al. (2007). Virtual reality exposure therapy for the treatment of posttraumatic stress disorder following September 11, 2001. J. Clin. Psychiatry 68, 1639-1647. doi: 10.4088/JCP.v68n1102

Difede, J., Cukor, J., Wyka, K., Olden, M., Hoffman, H., Lee, F. S., et al. (2013). D-cycloserine augmentation of exposure therapy for post-traumatic stress disorder: a pilot randomized clinical trial. Neuropsychopharmacology 39, 1052-1058. doi: 10.1038/npp.2013.317

Ecrepont, A., Haidon, C., Girard, B., and Menelas, B.-A. J. (2016). "A fully customizable truck-driving simulator for the care of people suffering from posttraumatic stress disorder," in 2016 IEEE International Conference on Serious Games and Applications for Health: May 11-13, Orlando, FL, USA (Piscataway, NJ: IEEE), $1-8$.

Foa, E., Hembree, E. A., Rauch, S., and Rothbaum, B. O. (2019). Prolonged Exposure Therapy for PTSD: Emotional Processing of Traumatic Experiences Therapist Guide. New York, NY: Oxford University Press.

Foa, E. B., Hembree, E., and Rothbaum, B. (2007). Prolonged Exposure Therapy for PTSD: Therapist Guide. New York, NY: Oxford University Press.

Foa, E. B., Hembree, E. A., Cahill, S. P., Rauch, S. A. M., Riggs, D. S., Feeny, N. C., et al. (2005). Randomized trial of prolonged exposure for posttraumatic stress disorder with and without cognitive restructuring: outcome at academic and community clinics. J. Consult. Clin. Psychol. 73, 953-964. doi: 10.1037/0022-006X.73.5.953

Foa, E. B., and Kozak, M. J. (1986). Emotional processing of fear. Exposure to corrective information. Psychol. Bull. 99, 20-35. doi: 10.1037/0033-2909.99.1.20

Foa, E. B., McLean, C. P., Zang, Y., Rosenfield, D., Yadin, E., Yarvis, J. S., et al. (2018). Effect of prolonged exposure therapy delivered over 2 weeks vs 8 weeks vs present-centered therapy on PTSD symptom severity in military personnel: a randomized clinical trial. JAMA 319, 354-364. doi: 10.1001/jama.2017.21242

Foa, E. B., and McNally, R. J. (1996). "Mechanisms of change in exposure therapy," in Current Controversies in the Anxiety Disorders, ed R. M. Rapee (New York, NY:L Guilford Press), 329-343.

Foa, E. B., Rothbaum, B. O., Riggs, D. S., and Murdock, T. B. (1991). Treatment of posttraumatic stress disorder in rape victims. A comparison between cognitivebehavioral procedures and counseling. J. Consult. Clin. Psychol. 59, 715-723. doi: 10.1037/0022-006X.59.5.715

Fonzo, G. A., Goodkind, M. S., Oathes, D. J., Zaiko, Y. V., Harvey, M., Peng, K. K., et al. (2017). PTSD psychotherapy outcome predicted by brain activation during emotional reactivity and regulation. Am. J. Psychiatry 174, 1163-1174. doi: 10.1176/appi.ajp.2017.16091072

Hartmann, T., Wirth, W., Schramm, H., Klimmt, C., Vorderer, P., Gysbers, A., et al. (2016). The spatial presence experience scale (SPES). J. Media Psychol. 28, 1-15. doi: $10.1027 / 1864-1105 / \mathrm{a} 000137$
Hedges, L. V., and Olkin, I. (2014). Statistical Method for Meta-Analysis. Burlington: Elsevier Science.

Hembree, E. A., Rauch, S. A., and Foa, E. B. (2003). Beyond the manual. The insider's guide to prolonged exposure therapy for PTSD. Cogn. Behav. Prac. 10, 22-30. doi: 10.1016/S1077-7229(03)80005-6

Högberg, G., Pagani, M., Sundin, O., Soares, J., Aberg-Wistedt, A., Tärnell, B., et al. (2007). On treatment with eye movement desensitization and reprocessing of chronic post-traumatic stress disorder in public transportation workers-a randomized controlled trial. Nord. J. Psychiatry 61, 54-61. doi: 10.1080/08039480601129408

Hundt, N. E., Ecker, A. H., Thompson, K., Helm, A., Smith, T. L., Stanley, M. A. et al. (2018). "It didn't fit for me:" A qualitative examination of dropout from prolonged exposure and cognitive processing therapy in veterans. Psychol. Serv. 15, 536-542. doi: 10.1037/ser0000316

Jaycox, L. H., Foa, E. B., and Morral, A. R. (1998). Influence of emotional engagement and habituation on exposure therapy for PTSD. J. Consult. Clin. Psychol. 66, 185-192. doi: 10.1037/0022-006X.66.1.185

Jetly, C. R., Meakin, L. C., Sinitski, E. H., Blackburn, L., Menard, J., Vincent, M., et al. (2017). Multi-modal virtual-reality based treatment for members with combat related posttraumatic stress disorder: Canadian Armed Forces pilot study. Virt. Rehabil. ICVR 2017, 1-2. doi: 10.1109/ICVR.2017.8007474

Karatzias, T., Power, K., Brown, K., McGoldrick, T., Begum, M., Young, J., et al. (2011). A controlled comparison of the effectiveness and efficiency of two psychological therapies for posttraumatic stress disorder: eye movement desensitization and reprocessing vs. emotional freedom techniques. J. Nervous Mental Dis. 199, 372-378. doi: 10.1097/NMD.0b013e31821cd262

Kehle-Forbes, S. M., Meis, L. A., Spoont, M. R., and Polusny, M. A. (2016). Treatment initiation and dropout from prolonged exposure and cognitive processing therapy in a VA outpatient clinic. Psychol. Trauma 8, 107-114. doi: $10.1037 /$ tra0000065

Kengne, S. A. K., Fossaert, M., Girard, B., and Menelas, B.-A. J. (2018). Actioncentered exposure therapy (ACET): a new approach to the use of virtual reality to the care of people with post-traumatic stress disorder. Behav. Sci. 8:76. doi: $10.3390 / \mathrm{bs} 8080076$

Koek, R. J., Schwartz, H. N., Scully, S., Langevin, J.-P., Spangler, S., Korotinsky, A., et al. (2016). Treatment-refractory posttraumatic stress disorder (TRPTSD): a review and framework for the future. Prog. Neuropsychopharmacol. Biol. Psychiatry 70, 170-218. doi: 10.1016/j.pnpbp.2016.01.015

Kothgassner, O. D., Goreis, A., Kafka, J. X., van Eickels, R. L., Plener, P. L., and Felnhofer, A. (2019). Virtual reality exposure therapy for posttraumatic stress disorder (PTSD): a meta-analysis. Eur. J. Psychotraumatol. 10:1654782. doi: 10.1080/20008198.2019.1654782

Leaman, S., Rothbaum, B., Difede, J., Cukor, J., Gerardi, M., and Rizzo, A. (2013). "Treating combat-related PTSD with virtual reality exposure therapy." in Handbook of Military Social Work, eds. A. Rubin, E. L. Weiss, and J. E. Coll (Hoboken, NJ: Wiley), 113-140.

Lee, K. M., Jung, Y., Kim, J., and Kim, S. R. (2006). Are physically embodied social agents better than disembodied social agents? The effects of physical embodiment, tactile interaction, and people's loneliness in human-robot interaction. Int. J. Hum. Comput. Stud. 64, 962-973. doi: 10.1016/j.ijhcs.2006.05.002

Lewis, C., Roberts, N. P., Gibson, S., and Bisson, J. I. (2020). Dropout from psychological therapies for post-traumatic stress disorder (PTSD) in adults: systematic review and meta-analysis. Eur. J. Psychotraumatol. 11:1709709. doi: 10.1080/20008198.2019.1709709

Ling, Y., Nefs, H. T., Morina, N., Heynderickx, I., and Brinkman, W.-P. (2014). A meta-analysis on the relationship between self-reported presence and anxiety in virtual reality exposure therapy for anxiety disorders. PLOS ONE 9:e96144. doi: 10.1371/journal.pone.0096144

Loucks, L., Yasinski, C., Norrholm, S. D., Maples-Keller, J., Post, L., Zwiebach, L., et al. (2018). You can do that?! Feasibility of virtual reality exposure therapy in the treatment of PTSD due to military sexual trauma. J. Anxiety Disord. 61, 55-63. doi: 10.1016/j.janxdis.2018.06.004

Luckerson, V. (2014). Facebook Buying Oculus Virtual-Reality Company for $\$ 2$ Billion. Available online at: https://time.com/37842/facebook-oculus-rift/ (accessed September 28, 2020).

Maples-Keller, J. L., Jovanovic, T., Dunlop, B. W., Rauch, S., Yasinski, C., Michopoulos, V., et al. (2018). When translational neuroscience fails in the 
clinic: dexamethasone prior to virtual reality exposure therapy increases dropout rates. J. Anxiety Disord. 61, 89-97. doi: 10.1016/j.janxdis.2018.10.006

Maples-Keller, J. L., Rauch, S. A. M., Jovanovic, T., Yasinski, C. W., Goodnight, J. M., Sherrill, A., et al. (2019). Changes in trauma-potentiated startle, skin conductance, and heart rate within prolonged exposure therapy for PTSD in high and low treatment responders. J. Anxiety Disord. 68:102147. doi: 10.1016/j.janxdis.2019.102147

Marcus, S. V., Marquis, P., and Sakai, C. (1997). Controlled study of treatment of PTSD using EMDR in an HMO setting. Psychotherapy 34, 307-315. doi: $10.1037 / \mathrm{h} 0087791$

Marks, I., Lovell, K., Noshirvani, H., Livanou, M., and Thrasher, S. (1998). Treatment of posttraumatic stress disorder by exposure and/or cognitive restructuring: a controlled study. Arch. Gen. Psychiatry 55, 317-325. doi: 10.1001/archpsyc.55.4.317

McDonagh, A., Friedman, M., McHugo, G., Ford, J., Sengupta, A., Mueser, K., et al. (2005). Randomized trial of cognitive-behavioral therapy for chronic posttraumatic stress disorder in adult female survivors of childhood sexual abuse. J. Consult. Clin. Psychol. 73, 515-524. doi: 10.1037/0022-006X.73.3.515

McLay, R. N., Baird, A., Webb-Murphy, J., Deal, W., Tran, L., Anson, H., et al. (2017). A randomized, head-to-head study of virtual reality exposure therapy for posttraumatic stress disorder. Cyberpsychol. Behav. Soc. Netw. 20, 218-224. doi: 10.1089/cyber.2016.0554

McLay, R. N., Wood, D. P., Webb-Murphy, J. A., Spira, J. L., Wiederhold, M. D., Pyne, J. M., et al. (2011). A randomized, controlled trial of virtual reality-graded exposure therapy for post-traumatic stress disorder in active duty service members with combat-related post-traumatic stress disorder. Cyberpsychol. Behav. Soc. Netw. 14, 223-229. doi: 10.1089/cyber.2011.0003

McLean, C. P., and Foa, E. B. (2011). Prolonged exposure therapy for posttraumatic stress disorder: a review of evidence and dissemination. Expert Rev. Neurother. 11, 1151-1163. doi: 10.1586/ern.11.94

Menelas, B.-A. J., Haidon, C., Ecrepont, A., and Girard, B. (2018). Use of virtual reality technologies as an action-cue exposure therapy for truck drivers suffering from post-traumatic stress disorder. Entertain. Comput. 24, 1-9. doi: 10.1016/j.entcom.2017.10.001

Mert, A., Buirma, R., van Luijk, J., Melkonian, G., Pels, J., and Vermetten, E. (2013). "Development of a theory-based applied game for the treatment of posttraumatic stress disorder: proof of feasibility," in Games for Health: Proceedings of the 3rd Conference on Gaming and Playful Interaction in Health Care, eds B. Schouten, S. Fedtke, T. Bekker, M. Schijven, and A. Gekker (Wiesbaden: Springer), 247-253.

Miyahira, S., Folen, R. A., Hoffman, H. G., Garcia-Palacios, A., Spira, J. L., and Kawasaki, M. (2012). The effectiveness of VR exposure therapy for PTSD in returning warfighters. Stud. Health Technol. Inform. 181, 123-132. doi: 10.3233/978-1-61499-121-2-128

National Institute for Health and Care Excellence (2018). Post-traumatic Stress Disorder. NICE Guideline [NG116]. Available online at: https://www.nice. org.uk/guidance/ng116/chapter/Recommendations\#management-of-ptsd-inchildren-young-people-and-adults (accessed September 28, 2020).

Nijdam, M. J., and Vermetten, E. (2018). Moving forward in treatment of posttraumatic stress disorder: innovations to exposure-based therapy. Eur. J. Psychotraumatol. 9:1458568. doi: 10.1080/20008198.2018.1458568

Nitsche, M. A., Cohen, L. G., Wassermann, E. M., Priori, A., Lang, N., Antal, A., et al. (2008). Transcranial direct current stimulation: state of the art 2008. Brain Stimul. 1, 206-223. doi: 10.1016/j.brs.2008.06.004

Oh, C., Herrera, F., and Bailenson, J. (2019). The effects of immersion and realworld distractions on virtual social interactions. Cyberpsychol. Behav. Soc. Netw. 22, 365-372. doi: 10.1089/cyber.2018.0404

Oh, C. S., Bailenson, J. N., and Welch, G. F. (2018). A systematic review of social presence. Definition, antecedents, and implications. Front. Robot. AI 5:114. doi: $10.3389 /$ frobt.2018.00114

Pacella, M. L., Armelie, A., Boarts, J., Wagner, G., Jones, T., Feeny, N., et al. (2011). The impact of prolonged exposure on PTSD symptoms and associated psychopathology in people living with HIV: a randomized test of concept. AIDS Behav. 16, 1327-1340. doi: 10.1007/s10461-011-0076-y

Peters, M. D. J., Godfrey, C. M., Khalil, H., McInerney, P., Parker, D., and Soares, C. B. (2015). Guidance for conducting systematic scoping reviews. Int. J. Evid. Based Healthc. 13, 141-146. doi: 10.1097/XEB.0000000000000050
Pham, M. T., Rajić, A., Greig, J. D., Sargeant, J. M., Papadopoulos, A., and McEwen, S. A. (2014). A scoping review of scoping reviews: advancing the approach and enhancing the consistency. Res. Synthesis Methods 5, 371-385. doi: $10.1002 /$ jrsm. 1123

Power, K., McGoldrick, T., Brown, K., Buchanan, R., Sharp, D., Swanson, V., et al. (2002). A controlled comparison of eye movement desensitization and reprocessing versus exposure plus cognitive restructuring versus waiting list in the treatment of post-traumatic stress disorder. Clin. Psychol. Psychother. 9, 299-318. doi: 10.1002/cpp.341

Powers, M. B., Halpern, J. M., Ferenschak, M. P., Gillihan, S. J., and Foa, E. B. (2010). A meta-analytic review of prolonged exposure for posttraumatic stress disorder. Clin. Psychol. Rev. 11, 428-436. doi: 10.1016/j.cpr.2010.04.007

Price, M., and Anderson, P. (2007). The role of presence in virtual reality exposure therapy. J. Anxiety Disord. 21, 742-751. doi: 10.1016/j.janxdis.2006.11.002

Rauch, S. A. M., King, A. P., Abelson, J., Tuerk, P. W., Smith, E., Rothbaum, B. O., et al. (2014). Biological and symptom changes in posttraumatic stress disorder treatment: a randomized clinical trial. Depress. Anxiety 32, 204-212. doi: $10.1002 / \mathrm{da} .22331$

Ready, D. J., Gerardi, R. J., Backscheider, A. G., Mascaro, N., and Rothbaum, B. O. (2010). Comparing virtual reality exposure therapy to present-centered therapy with 11 U.S. Vietnam veterans with PTSD. Cyberpsychol. Behav. Soc. Netw. 13, 49-54. doi: 10.1089/cyber.2009.0239

Reger, G. M., Koenen-Woods, P., Zetocha, K., Smolenski, D. J., Holloway, K. M., Rothbaum, B. O., et al. (2016). Randomized controlled trial of prolonged exposure using imaginal exposure vs. virtual reality exposure in active duty soldiers with deployment-related posttraumatic stress disorder (PTSD). J. Consul. Clin. Psychol. 84, 946-959. doi: 10.1037/ccp0000134

Ridout, S., Spofford, C., van't Wout, M., Unger, W., Philip, N., and Shea, M. T. (2017). Heart rate variability responses to a standardized virtual reality exposure in Veterans with PTSD. Biol. Psychiatry 81:S271. doi: 10.1016/j.biopsych.2017.02.1079

Rizzo, A., Roy, M. J., Hartholt, A., Costanzo, M., Highland, K. B., Jovanovic, T., et al. (2017). "Virtual reality applications for the assessment and treatment of PTSD," in Handbook of Military Psychology: Clinical and Organizational Practice, eds S. V. Bowles and P. T. Bartone (Cham: Springer), 453-471.

Rizzo, A. S., and Koenig, S. T. (2017). Is clinical virtual reality ready for primetime? Neuropsychology 31, 877-899. doi: 10.1037/neu0000405

Rizzo, A. S., and Shilling, R. (2017). Clinical virtual reality tools to advance the prevention, assessment, and treatment of PTSD. Eur. J. Psychotraumatol. 8:1414560. doi: 10.1080/20008198.2017.1414560

Rizzo, A., Pair, J., McNerney, P. S., Eastlund, E., Manson, B., Gratch, J., et al. (2005). Development of a VR therapy application for Iraq war military personnel with PTSD. Stud. Health Technol. Inform. 111, 407-413.

Rothbaum, B. O., Hodges, L., Alarcon, R., Ready, D., Shahar, F., Graap, K., et al. (1999). Virtual reality exposure therapy for PTSD Vietnam veterans: a case study. J. Trauma. Stress 12, 263-271. doi: 10.1023/A:1024772 308758

Rothbaum, B. O., Hodges, L. F., Ready, D., Graap, K., and Alarcon, R. D. (2001). Virtual reality exposure therapy for Vietnam veterans with posttraumatic stress disorder. J. Clin. Psychiatry 62, 617-622. doi: 10.4088/JCP.v62n0808

Rothbaum, B. O., Price, M., Jovanovic, T., Norrholm, S. D., Gerardi, M., Dunlop, B., et al. (2014). A randomized, double-blind evaluation of D-cycloserine or Alprazolam combined with virtual reality exposure therapy for posttraumatic stress disorder (PTSD) in Iraq and Afghanistan war veterans. Am. J. Psychiatry 171, 640-648. doi: 10.1176/appi.ajp.2014.13121625

Rothbaum, B. O., Rizzo, A. S., and Difede, J. (2010). Virtual reality exposure therapy for combat-related posttraumatic stress disorder. Ann. N. Y. Acad. Sci. 1208, 126-132. doi: 10.1111/j.1749-6632.2010.05691.x

Schäfer, I., Gast, U., Hofmann, A., Knaevelsrud, C., Lampe, A., Liebermann, P., et al. (2019). S3-Leitlinie Posttraumatische Belastungsstörung. Berlin: Springer.

Schmucker, M., and Köster, R. (2019). Praxishandbuch IRRT: Imagery Rescripting \& Reprocessing Therapy bei Traumafolgestörungen, Angst, Depression und Trauer. Vierte Auflage. Stuttgart: Klett-Cotta.

Schnurr, P. P., Friedman, M. J., Engel, C. C., Foa, E. B., Shea, M. T., Chow, B. K., et al. (2007). Cognitive behavioral therapy for posttraumatic stress disorder in women: a randomized controlled trial. JAMA 297, 820-830. doi: 10.1001/jama.297.8.820 
Shapiro, F. (1989). Efficacy of the eye movement desensitization procedure in the treatment of traumatic memories. J. Trauma. Stress 2, 199-223. doi: $10.1002 /$ jts. 2490020207

Shapiro, F. (2018). Eye Movement Desensitization and Reprocessing (EMDR) Therapy, Third Edition: Basic Principles, Protocols, and Procedures. New York, NY: Guilford Publications.

Sherman, W. R., and Craig, A. B. (2019). Understanding Virtual Reality: Interface, Application, and Design, 2nd Edn. Cambridge, MA: Morgan Kaufmann.

Shulman, G. P., Buck, B. E., Gahm, G. A., Reger, G. M., and Norr, A. M. (2019). Effectiveness of the intent to complete and intent to attend intervention to predict and prevent posttraumatic stress disorder treatment drop out among soldiers. J. Trauma Stress 32, 784-790. doi: 10.1002/jts.22427

Slater, M. (2009). Place illusion and plausibility can lead to realistic behaviour in immersive virtual environments. Philos. Trans. R Soc. Lond. B. Biol. Sci. 364, 3549-3557. doi: 10.1098/rstb.2009.0138

Slater, M., and Wilbur, S. (1997). A framework for immersive virtual environments (FIVE). Speculations on the role of presence in virtual environments. Pres. Teleoperat. Virtual Environ. 6, 603-616. doi: 10.1162/pres.1997.6.6.603

Steenkamp, M. M., Litz, B. T., Hoge, C. W., and Marmar, C. R. (2015). Psychotherapy for military-related PTSD: a review of randomized clinical trials. JAMA 314, 489-500. doi: 10.1001/jama.2015.8370

Taylor, S., Thordarson, D. S., Maxfield, L., Fedoroff, I. C., Lovell, K., and Ogrodniczuk, J. (2003). Comparative efficacy, speed, and adverse effects of three PTSD treatments: exposure therapy, EMDR, and relaxation training. J. Consult. Clin. Psychol. 71, 330-338. doi: 10.1037/0022-006X.71.2.330

Tricco, A. C., Lillie, E., Zarin, W., O’Brien, K. K., Colquhoun, H., Levac, D., et al. (2018). PRISMA extension for scoping reviews (PRISMA-ScR): checklist and explanation. Ann. Intern. Med. 169, 467-473. doi: 10.7326/M18-0850

Turner, S. M., Beidel, D. C., and Frueh, B. C. (2005). Multicomponent behavioral treatment for chronic combat-related posttraumatic stress disorder: trauma management therapy. Behav. Modif. 29, 39-69. doi: 10.1177/0145445504270872

Van Gelderen, M. J., Nijdam, M. J., Haagen, J. F. G., and Vermetten, E. (2020). Interactive motion-assisted exposure therapy for veterans with treatment-resistant posttraumatic stress disorder: a randomized controlled trial. Psychother. Psychosom. 89, 215-227. doi: 10.1159/000505977

Van Gelderen, M. J., Nijdam, M. J., and Vermetten, E. (2018). An innovative framework for delivering psychotherapy to patients with treatment-resistant posttraumatic stress disorder: rationale for interactive motion-assisted therapy. Front. Psychiatry 9:176. doi: 10.3389/fpsyt.2018.00176
Van Minnen, A., Harned, M. S., Zoellner, L., and Mills, K. (2012). Examining potential contraindications for prolonged exposure therapy for PTSD. Eur. J. Psychotraumatol. 3:18805. doi: 10.3402/ejpt.v3i0.18805

Van't Wout, M., Shea, M. T., Larson, V. C., Greenberg, B. D., and Philip, N. S. (2018). Combined transcranial direct current stimulation with virtual reality exposure for posttraumatic stress disorder: feasibility and pilot results. Brain Stimul. 12, 41-43. doi: 10.1016/j.brs.2018.09.011

Van't Wout, M., Spofford, C. M., Unger, W. S., Sevin, E. B., and Shea, M. T. (2017). Skin conductance reactivity to standardized virtual reality combat scenes in veterans with PTSD. Appl. Psychophysiol. Biofeedback 42, 209-221. doi: 10.1007/s10484-017-9366-0

Vermetten, E., Meijer, L., van der Wurff, P., and Mert, A. (2013). The effect of military motion-assisted memory desensitization and reprocessing treatment on the symptoms of combat-related post traumatic stress disorder: first preliminary results. Ann. Rev. Cyberther. Telemed. 191, 125-127. doi: 10.3233/978-1-61499-282-0-125

Wagenmans, A., van Minnen, A., Sleijpen, M., and Jongh, A., de (2018). The impact of childhood sexual abuse on the outcome of intensive trauma-focused treatment for PTSD. Eur. J. Psychotraumatol. 9:1430962. doi: 10.1080/20008198.2018.1430962

Wirth, W., Hartmann, T., Böcking, S., Vorderer, P., Klimmt, C., Schramm, H., et al. (2007). A process model of the formation of spatial presence experiences. Media Psychol. 9, 493-525. doi: 10.1080/15213260701283079

Zoellner, L. A., Feeny, N. C., Bittinger, J. N., Bedard-Gilligan, M. A., Slagle, D. M., Post, L. M., et al. (2011). Teaching trauma-focused exposure therapy for PTSD: critical clinical lessons for novice exposure therapists. Psychol. Trauma 3, 300-308. doi: 10.1037/a0024642

Conflict of Interest: The authors declare that the research was conducted in the absence of any commercial or financial relationships that could be construed as a potential conflict of interest.

Copyright (C) 2020 Knaust, Felnhofer, Kothgassner, Höllmer, Gorzka and Schulz. This is an open-access article distributed under the terms of the Creative Commons Attribution License (CC BY). The use, distribution or reproduction in other forums is permitted, provided the original author(s) and the copyright owner(s) are credited and that the original publication in this journal is cited, in accordance with accepted academic practice. No use, distribution or reproduction is permitted which does not comply with these terms. 